\title{
Disease Modeling with Human Neurons Reveals LMNB1 Dysregulation Underlying DYT1 Dystonia
}

\author{
Baojin Ding, ${ }^{1,2 *}$ Yu Tang, ${ }^{1,3 *}{ }^{\circledR}$ Shuaipeng Ma, ${ }^{1}$ Masuma Akter, ${ }^{2}$ Meng-Lu Liu, ${ }^{1}$ Tong Zang, ${ }^{1}$ and \\ Chun-Li Zhang ${ }^{1}$ \\ ${ }^{1}$ Department of Molecular Biology, Hamon Center for Regenerative Science and Medicine, University of Texas Southwestern Medical Center, Dallas, \\ Texas 75390, ${ }^{2}$ Department of Biology, University of Louisiana at Lafayette, Lafayette, Louisiana 70503, and ${ }^{3}$ National Clinical Research Center for \\ Geriatric Disorders, Department of Geriatrics, Xiangya Hospital, Central South University, Changsha, Hunan Province 410008, China
}

DYT1 dystonia is a hereditary neurologic movement disorder characterized by uncontrollable muscle contractions. It is caused by a heterozygous mutation in Torsin A (TOR1A), a gene encoding a membrane-embedded ATPase. While animal models provide insights into disease mechanisms, significant species-dependent differences exist since animals with the identical heterozygous mutation fail to show pathology. Here, we model DYT1 by using human patient-specific cholinergic motor neurons (MNs) that are generated through either direct conversion of patients' skin fibroblasts or differentiation of induced pluripotent stem cells (iPSCs). These human MNs with the heterozygous TOR1A mutation show reduced neurite length and branches, markedly thickened nuclear lamina, disrupted nuclear morphology, and impaired nucleocytoplasmic transport (NCT) of mRNAs and proteins, whereas they lack the perinuclear "blebs" that are often observed in animal models. Furthermore, we uncover that the nuclear lamina protein LMNB1 is upregulated in DYT1 cells and exhibits abnormal subcellular distribution in a cholinergic MNs-specific manner. Such dysregulation of LMNB1 can be recapitulated by either ectopic expression of the mutant TOR1A gene or shRNA-mediated downregulation of endogenous TOR1A in healthy control MNs. Interestingly, downregulation of LMNB1 can largely ameliorate all the cellular defects in DYT1 MNs. These results reveal the value of disease modeling with human patient-specific neurons and indicate that dysregulation of LMNB1, a crucial component of the nuclear lamina, may constitute a major molecular mechanism underlying DYT1 pathology.

Key words: cholinergic motor neurons; dystonia; human neurons; nuclear LMNB1; nucleocytoplasmic transport; TOR1A

Significance Statement

Inaccessibility to patient neurons greatly impedes our understanding of the pathologic mechanisms for dystonia. In this study, we employ reprogrammed human patient-specific motor neurons (MNs) to model DYT1, the most severe hereditary form of dystonia. Our results reveal disease-dependent deficits in nuclear morphology and nucleocytoplasmic transport (NCT). Most importantly, we further identify LMNB1 dysregulation as a major contributor to these deficits, uncovering a new pathologic mechanism for DYT1 dystonia.

Received Sep. 24, 2020; revised Dec. 23, 2020; accepted Dec. 30, 2020.

Author contributions: B.D., Y.T., and C.-L.Z. designed research; B.D., Y.T., S.M., M.A., and T.Z. performed research; B.D. and M.-L.L. contributed unpublished reagents/analytic tools; B.D., Y.T., S.M., M.A., and T.Z. analyzed data; B.D., Y.T., and C.-L.Z. wrote the paper.

This work was supported by The Welch Foundation Grant I-1724, Texas Alzheimer's Research Consortium (TARCC), The Decherd Foundation, The Pape Adams Foundation, the Kent Waldrep Foundation Center for Basic Research on Nerve Growth and Regeneration, National Institutes of Health (NIH) Grants NS092616, NS099073, NS111776, and NS117065 (to C.-L.Z.) and NS112910 (to B.D.), Friends of the Alzheimer's Disease Center (ADC), the NIH ADC Grant NIH/ NIA P30-12300-21 (to B.D.), the Department of Defense Peer Reviewed Medical Research Program (PRMRP) Grant W81XWH2010186 (to B.D.), the National Natural Sciences Foundation of China Grant No. 81801200 (to Y.T.), and the Hunan Provincial Natural Science Foundation of China Grant No. 2019 Jj40476 (to Y.T.). We thank members of the Zhang laboratory (Xiaoling Zhong, Wenjiao Tai, Leilei Wang, Yunjia Zhang, and Yuhua Zou) and the Ding laboratory (Masood Sepehrimanesh, Haochen Cui, and Jacob A. Stagray) for technical help and Dr. William T. Dauer (University of Texas Southwestern) for discussion. We also thank the support from the University of Texas Southwestern Electron Microscopy Core and the Microscopy Center at the University of Louisiana at Lafayette.

*B.D. and Y.T. contributed equally to this work. The author order was determined by workload and leadership in the project.

The authors declare no competing financial interests.

Correspondence should be addressed to Baojin Ding Baojin.Ding@Louisiana.edu or Chun-Li Zhang at chun-li.zhang@utsouthwestern.edu.

https://doi.org/10.1523/JNEUROSCI.2507-20.2020

Copyright $\odot 2021$ the authors

\section{Introduction}

Dystonia is a common movement disorder characterized by sustained or intermittent muscle contractions causing abnormal movements and/or postures (Albanese et al., 2013). The clinical characteristics and underlying causes of dystonia are very heterogeneous, so the cellular mechanism for dystonia remains elusive (Lohmann and Klein, 2013). Childhood-onset Torsin dystonia, also called DYT1 dystonia, represents the most frequent and severe form of hereditary isolated dystonia (Albanese et al., 2013; Dauer, 2014). Most cases of typical DYT1 dystonia are caused by a heterozygous 3-bp deletion ( $\triangle \mathrm{GAG}$ ) in exon 5 of the TOR $1 A$ gene, resulting in the loss of one of the two adjacent glutamate residues (E302 or E303) in the carboxy-terminal region of Torsin A protein (TOR1A $\Delta \mathrm{E}$; Ozelius et al., 1997; Charlesworth et al., 2013).

Protein TOR1A belongs to the superfamily of AAA+ (ATPases associated with diverse cellular activities) proteins (Ozelius et al., 1997). It is widely distributed throughout the 
human CNS (Rostasy et al., 2003). Most TOR1A proteins are embedded in the lumen of the endoplasmic reticulum and the endomembrane space of the nuclear envelope (NE; Vander Heyden et al., 2011). Accumulating evidence indicates that TOR1A plays an important role in membrane-cytoskeleton interactions (Laudermilch and Schlieker, 2016). At the NE, TOR1A is associated with the linkage between NE and cytoskeleton via binding the KASH domain of nesprins (Nery et al., 2008). It is also involved in the regulation of NE membrane morphology (Naismith et al., 2004; Goodchild et al., 2005; Laudermilch et al., 2016), the localization and function of nuclear pore subunits (VanGompel et al., 2015), and the nuclear egress of large ribonucleoprotein granules (Jokhi et al., 2013). Furthermore, TOR1A regulates neurite extension (Hewett et al., 2006) and synaptic vesicle recycling (Granata et al., 2008), underscoring its critical functions in neuronal development and function.

Although TOR1A $\triangle \mathrm{E}$ has been shown to be a loss-of-function mutation (Torres et al., 2004; Goodchild et al., 2005; Zhao et al., 2013), the cellular mechanisms underlying DYT1 dystonia remain largely unknown. Traditionally, dystonia is considered as a disorder of the basal ganglia (Tewari et al., 2017), supported by the findings in Torla-mutant mice including the early structural and functional plasticity alterations in the corticostriatal synapses (Maltese et al., 2018) and the dysregulation of the striatal dopaminergic neutrons (Ip et al., 2016). The cerebellum via direct modulation of the basal ganglia is also shown as a major site of dysfunction in Torla knock-out mice (Fremont et al., 2017; Tewari et al., 2017). One cellular hallmark in Torla knock-out mice is the abnormal NE morphology in multiple areas of the CNS including the large ventral horn of the spinal cord (Goodchild et al., 2005), indicating that the lower motor neurons (MNs) might be particularly affected. Unlike the majority of human DYT1 patients who are affected by the heterozygous TOR1A mutation, mice with the identical mutation as a heterozygote nonetheless failed to exhibit any pathologic phenotypes (Goodchild et al., 2005), clearly indicating there might be species-dependent differences on disease mechanisms. However, DYT1 has never been previously modeled in human neurons.

Through cell fate reprogramming, we successfully generated human cholinergic MNs from fibroblasts of DYT1 patients and healthy controls via two strategies: direct conversion (diMNs) and differentiation through induced pluripotent stem cells (iPSCs; iMNs). We found that DYT1 neurons generated by both methods show impairments in neuron development, NE morphology, mRNA nuclear export, and protein transport. We further discovered that nuclear lamin B1 (LMNB1) is dysregulated in both expression and subcellular localization in patient MNs. Importantly, LMNB1 downregulation can largely ameliorate defects of NE morphology and nuclear transport in patient neurons. These findings indicate that LMNB1 dysregulation may contribute to DYT1 dystonia and provide a novel molecular target for intervention.

\section{Materials and Methods}

Cell lines and culture condition

HEK 293T cells (CRL-11268) and SH-SY5Y (CRL-11266) cells were purchased from ATCC. DYT1 fibroblast and healthy controls, GM03211 (DYT1-1, male, 30 years old), NDS00305 (DYT1-2, male, 22 years old), GM02304 (DYT1-3, female, 17 years old), NDS00301 (DYT1-4, female, 53 years old), GM00024 (Control-1, male, 31 years old), GM03652 (Control-2, male, 24 years old), GM04506 (Control-3, female, 20 years old), and AG07473 (Control-4, female, 50 years old) were obtained from the Coriell Institute for Medical Research or NINDS Human Cell and Data Repository (NHCDR). Human age-matched wild-type (WT) iPSC (GM23476) was obtained from Coriell and H9 embryonic stem cell (ESC) line (WA09) was purchased from WiCell Research Institute. Human iPSCs were maintained in complete mTeSR1 medium (STEMCELL Technologies) on Matrigel (Corning) coated dishes at $37^{\circ} \mathrm{C}$ and $5 \% \mathrm{CO}_{2}$, and the medium was daily replaced.

The medium recipes were as following:

(1) HEK medium: DMEM supplemented with $10 \%$ fetal bovine serum (FBS) and $1 \%$ penicillin/streptomycin (P/S).

(2) Fibroblast medium: DMEM supplemented with 15\% FBS and 1\% $\mathrm{P} / \mathrm{S}$.

(3) Neuronal induction medium: DMEM:F12:neurobasal (2:2:1), $0.8 \% \mathrm{~N} 2$ (Invitrogen), 0.8\% B27 (Invitrogen), 1\% P/S, and supplemented with $10 \mathrm{~mm}$ Forskolin (FSK, Sigma-Aldrich), $1 \mathrm{~mm}$ dorsomorphin (DM; Millipore), and $10 \mathrm{ng} / \mathrm{ml}$ basic fibroblast growth factor (bFGF; PeproTech).

(4) Neuronal maturation medium: DMEM:F12:neurobasal (2:2:1), $0.8 \% \mathrm{~N} 2$ (Invitrogen), $0.8 \% \mathrm{~B} 27$ (Invitrogen), $1 \% \mathrm{P} / \mathrm{S}$, and supplemented with $5 \mathrm{~mm}$ FSK and $10 \mathrm{ng} / \mathrm{ml}$ each of BDNF, GDNF, and NT3 (PeproTech).

(5) ESC medium: DMEM/F12 medium with 20\% KnockOut Serum Replacement (KOSR; Thermo Fisher Scientific), 1\% GlutaMax, $1 \%$ non-essential amino acid (NEAA), $50 \mu \mathrm{M} \beta$-mercaptoethanol ( $\beta$-ME), $1 \% \mathrm{P} / \mathrm{S}$, and $10 \mathrm{ng} / \mathrm{ml}$ bFGF (PeproTech).

(6) Neurosphere medium (NSP medium): DMEM/F12 medium containing $1 \% \mathrm{~N} 2,1 \%$ GlutaMax, $1 \%$ NEAA, $50 \mu \mathrm{M} \beta$-ME, $1 \% \mathrm{P} / \mathrm{S}$, $8 \mu \mathrm{g} / \mathrm{ml} \mathrm{Heparin,} 20 \mathrm{ng} / \mathrm{ml} \mathrm{bFGF}$, and $20 \mathrm{ng} / \mathrm{ml}$ epidermal growth factor (EGF; PeproTech).

(7) Neural progenitor cell medium: DMEM/F12 and neurobasal medium (1:1) containing $0.5 \% \quad \mathrm{~N} 2 \quad$ (Invitrogen), $\quad 1 \% \quad \mathrm{~B} 27$ (Invitrogen), $1 \%$ GlutaMax, $1 \%$ NEAA, $50 \mu \mathrm{M} \beta$-ME, $1 \% \mathrm{P} / \mathrm{S}$, $10 \mathrm{ng} / \mathrm{ml} \mathrm{EGF}$ and $10 \mathrm{ng} / \mathrm{ml} \mathrm{bFGF}$.

(8) SH-SY5Y medium: DMEM supplemented with $15 \%$ FBS and $1 \%$ $\mathrm{P} / \mathrm{S}$.

(9) SH-SY5Y differentiation medium: DMEM supplemented with $3 \% \mathrm{FBS}, 10 \mu \mathrm{M}$ all-trans-retinoic acid (RA; Sigma) and 1\% P/S

Plasmid construction and virus production

A third-generation lentiviral vector ( $p C S C$-SP-PW-IRES-GFP) was used to express NEUROG2-IRES-GFP-T2A-Sox11, NEUROG2-IRES-Sox11, or ISL1-T2A-LHX3. cDNAs for TOR1A and TOR1A $\triangle E$ were provided by Gonzalo E. Torres (Torres et al., 2004) and were individually subcloned into $p C S C$-SP-PW-IRES-GFP vector at the BamHI and XhoI restriction sites. Another third-generation lentiviral vector ( $L V$-CAG-mCherrymiRE-Luc) was used to express TOR1A-shRNAs or LMNB1-shRNAs as described in previously report (Pelossof et al., 2017). Briefly, shRNA oligos targeting the gene coding regions were synthesized, PCR amplified with flanking miR-30 sequences, and ligated into the above lentiviral vector at the XhoI and EcoRI restriction sites. The targeting sequences are AACGGTGTTCACCAAGTTAGAT (TOR1AshRNA-1), CAGCAAGATCATCGCAGAGAATA (TOR1A-shRNA-2), AGCTTCTTGATGTAAAGTTA (LMNB1-shRNA-1), or CAGACTGT CATCAGAGATGAA (LMNB1-shRNA-2). All plasmids were verified by restriction enzyme digestions and DNA sequencing. The dual reporter 2Gi2R was generously provided by Fred H. Gage (Mertens et al., 2015). The Moloney murine leukemia virus-based retroviral vectors (pMXs) carrying OCT4, SOX2, KLF4, or c-MYC were obtained from Addgene (Takahashi et al., 2007). Each of these vectors was co-transfected with packaging plasmids ( $p C M V$-Gag-Pol and $p C M V$-VSVG) into HEK293T cells for retrovirus production. Replication-incompetent lentiviruses were produced and viral supernatants were collected at 48 and $72 \mathrm{~h}$ posttransfection (Ding and Kilpatrick, 2013). The viral supernatants were filtered through $0.45-\mu \mathrm{m}$ syringe filters and stored at $4^{\circ} \mathrm{C}$ before cell transduction.

\section{Direct conversion of adult fibroblasts into MNs}

Lentiviral delivery of four factors (NEUROG2, Sox11, ISL1, and LHX3) was used as previously described (Liu et al., 2016; Tang et al., 2017; Ding et al., 2020). In brief, fibroblasts were plated at a density of $1 \times 10^{4}$ cells/ 
$\mathrm{cm}^{2}$ onto Matrigel-coated dishes. Cells were transduced the next day with lentiviral supernatants supplemented with $6 \mathrm{mg} / \mathrm{ml}$ polybrene. Fibroblast medium was refreshed after overnight incubation. One day later, the culture was replaced with neuronal induction medium and half-changed every other day until $14 \mathrm{~d}$ postinfection (dpi). A replating procedure (Liu et al., 2013) was used to purify induced neurons. The purified cells were cultured in neuronal maturation medium onto Matrigel-coated coverslips with or without the presentence of astrocytes depending on desired experiments. The medium was half changed twice a week until analysis.

\section{DYT1 iPSC generation}

DYT1 fibroblasts were plated into gelatin-coated $10 \mathrm{~cm}$ dishes $\left(8 \times 10^{5}\right.$ cells/dish) and transduced with a cocktail of four retroviruses (1:1:1:1) expressing OCT4, SOX2, KLF4, and c-MYC. Two days later, fibroblasts received a second round of virus infection and was marked as day 0 . On day 5 , cells were replated with fibroblast medium at $2 \times 10^{5}$ cells per well in Matrigel-coated six-well plates. Culture medium was replaced with human ESC medium on day 6, and then changed to mTeSR1 medium in the following days. Cells were treated with $0.5 \mathrm{~mm}$ VPA (Sigma) for $10 \mathrm{~d}$ starting on day 5. Based on ESC-like colony morphology, iPSC colonies were picked around three to four weeks. The picked colonies were then expanded and maintained in Matrigel-coated plates with mTeSR1 medium.

\section{Neural progenitor cells and neuronal differentiation}

Neural progenitor cells were generated as previously reported with minor modifications (Yang et al., 2014). Briefly, iPSCs or ESCs were cultured in mTeSR1 medium with $10 \mu \mathrm{m}$ RA and $0.5 \mathrm{~mm}$ VPA in Matrigelcoated $6-\mathrm{cm}$ plates for $7 \mathrm{~d}$. Cells were then digested with Versene and gently pipetted into small clumps supplemented with $10 \mu \mathrm{M}$ Y-27632. Cell clumps were aggregated in KOSR medium for $4 \mathrm{~d}$, followed by culturing in NSP medium for another week. The resulting neurospheres were then dissociated into single cells with Accutase (Innovative Cell Technologies), and maintained in neural progenitor cell medium. For MN differentiation (Tang et al., 2017; Sepehrimanesh and Ding, 2020), neural progenitor cells were plated into Matrigel-coated plates at a density of $3 \times 10^{4}$ cells $/ \mathrm{cm}^{2}$ and transduced with a cocktail of two lentiviruses (1:1) expressing NEUROG2-IRES-GFP-T2A-Sox11 and ISL1-T2A$L H X 3$. Culture medium was replaced the next day with neuronal maturation medium. Neurons were dissociated with Accutase on day 6 and replated onto astrocyte-coated coverslips for long-term culture.

\section{Immunostaining, confocal microscopy, and image analysis}

Cultured cells at the indicated time points were fixed with $4 \%$ paraformaldehyde (PFA) in PBS for $15 \mathrm{~min}$ at room temperature (RT) and then permeabilized and blocked for $1 \mathrm{~h}$ in blocking buffer (PBS containing $0.2 \%$ Triton X-100 and 3\% BSA). They were subsequently incubated overnight with primary antibodies in blocking buffer at $4^{\circ} \mathrm{C}$, followed by washing and incubation with corresponding fluorophoreconjugated secondary antibodies. Cell nuclei were counterstained with Hoechst 33342 (HST; Thermo Fisher Scientific). Primary antibodies used in this study were choline acetyltransferase (CHAT; Millipore, AB144P, 1:200), GFP (Aves, GFP-1020, 1:1000), HB9 (DSHB, 81.5C10, 1:500), lamin A (LMNA)/LMNC (Abcam, ab40567, 1:300), LMNB1 (Proteintech, 12 987-1-AP, 1:500), MAB414 (BioLegend, 902901, 1:500), MAP2 (Sigma, M4403, 1:500), MAP2 (Abcam, ab5392, 1:10,000), NUP98 (Santa Cruz, sc-74 578, 1:200), NUP359 (Millipore, ABN1385, 1:100), SMI32 (BioLegend, 801701, 1:5000), SYN1 (Cell Signaling, 5297, 1:200), TUBB3 (Covance, MMS-435P, 1:2000), and TUBB3 (Covance, PRB-435P-100, 1:2000).

Confocal images were obtained with a Nikon A1R or Zeiss LSM700 $(63 \times / 1.4 \mathrm{NA})$ confocal microscope. Neurite length was measured with the ImageJ software and shown as relative length per neurite (Ding et al., $2013,2016,2018)$. Neurites with direct connections to the soma were counted and shown as primary branches per cell. For nuclear morphology assay, we zoomed in on individual nucleus by using the ImageJ software and defined the abnormal nucleus based on two criteria: (1) at least one obvious sharp angle or a deep invagination; and (2) at least half area of the nucleus losing the smooth outline. Signals of nuclei (HST) or nuclear lamins were used to distinguish the nucleus and cytoplasm.

\section{Fluorescent in situ hybridization (FISH)}

FISH was performed as described previously (Ding et al., 2020). Briefly, cultured cells were washed once with PBS, and fixed with 4\% PFA for $30 \mathrm{~min}$ at RT. Cells were permeabilized with $0.2 \%$ Triton X-100 for $10 \mathrm{~min}$, followed by two washes with PBS. Samples were equilibrated with hybridization buffer composed of $2 \times$ SSC, $10 \%$ dextran sulfate, 10 mM ribonucleoside-vanadyl complex (RVC; New England Biolabs) and $20 \%$ formamide. A mixture of digoxigenin (DIG)-labeled oligo-dT or dA probes $(0.2 \mathrm{ng} / \mu \mathrm{l})$ and yeast tRNA $(0.2 \mu \mathrm{g} / \mu \mathrm{l})$ were heated to $95^{\circ} \mathrm{C}$ for $5 \mathrm{~min}$ and immediately chilled on ice. Probes were then combined with equal volumes of $2 \times$ hybridization buffer and incubated with samples overnight at $37^{\circ} \mathrm{C}$. Anti-DIG antibody (Sigma, 11333089001 , $1: 100)$ and corresponding fluorophore-conjugated secondary antibodies (Thermo Fisher Scientific) were used to detect oligo-dT signals. Anti-MAP2 (Abcam, ab5392, 1:10,000) antibody and HST were used to determine neuronal soma and nuclei, respectively. Fluorescence density of confocal images were analyzed with the ImageJ software. The "mean" values of the nucleus and the cytoplasm in each neuron were used to calculate the ratio of dT signal in nucleus to cytoplasm (Ding et al., 2020). We measured as large an area as possible for each neuron and employed an unbiased approach for data collection. The person who analyzed the images was completely blinded to the sample information.

\section{Western blot analysis}

Cells were lysed in lysis buffer composed of $50 \mathrm{~mm}$ Tris- $\mathrm{HCl}$ buffer $(\mathrm{pH}$ 8.0), $150 \mathrm{~mm} \mathrm{NaCl}, 1 \% \mathrm{NP} 40,1 \%$ Triton X-100, 0.1\% SDS, $0.5 \%$ sodium deoxycholate, and protease inhibitor cocktail (Roche). Equal amounts of cell lysates $(20 \mu \mathrm{g}$ per lane) were used for SDS-PAGE and western blot analysis as previously described (Ding et al., 2018). Primary antibodies and dilutions were Actin (Sigma, A5441, 1:60,000), TOR1A (Cell Signaling, 2150, 1;1000), TUBB3 (Covance, MMS-435P, 1:10,000), LMNB1 (Proteintech, 12 987-1-AP, 1:2000), LMNB2 (Abcam, ab97513 1:1000), GLE1 (Abcam, ab96007, 1:2000), LAP2A (Abcam, ab5162, 1:2000), LAP2B (Thermo Fisher Scientific, A304-840A, 1:2000), and RANGAP1 (Cell Signaling, 36067, 1:1000).

\section{Quantitative real-time PCR analysis}

Total RNA was extracted from cultured cells using TRIzol (Life Technologies), and genomic contamination was removed using TURBO DNase (Life Technologies). cDNA synthesis reactions were performed using $0.5 \mu \mathrm{g}$ of RNA from each sample with the SuperScriptIII First-Strand kit (Life Technologies) and random hexamer primers. Real-time PCR was performed in triplicate using primers, SYBR GreenER SuperMix (Invitrogen), and the ABI 7900HT thermocycler (Applied Biosystems). Target mRNA levels were normalized to the reference gene HPRT by $2^{-\Delta \Delta \mathrm{Ct}}$ method as described previously (Ding et al., 2013, 2016, 2018). PCR primer sequences (from 5' to 3') were CTGGTGGATGGGGTCTCCTA (qTOR1A-F), GAC ACAGACAACGCGTGTTC (qTOR1A-R), AGGAAAGCGGAAGAGGG TTG (qLMNB1-F), and GCCTCCCATTGGTTGATCCT (qLMNB1-R).

\section{Transmission electron microscopy (TEM)}

Cells on Matrigel-coated glass coverslips were fixed overnight at $4^{\circ} \mathrm{C}$ with a solution composed of $4 \%$ PFA, $1 \%$ glutaraldehyde, $1.5 \mu \mathrm{M} \mathrm{MgCl}_{2}$ and $5 \mu \mathrm{M} \mathrm{CaCl}_{2}$ in $0.1 \mathrm{M}$ cacodylate buffer ( $\mathrm{pH}$ 7.4). Samples were then twice washed with cacodylate buffer, treated for $1.5 \mathrm{~h}$ at RT with $1 \%$ osmium tetraoxide $\left(\mathrm{OsO}_{4} ; \mathrm{EMS}\right)$ and $0.8 \% \mathrm{~K} 3 \mathrm{Fe}(\mathrm{CN} 6)$ in cacodylate buffer, and prestained with $2 \%$ uranyl acetate in water for $2 \mathrm{~h}$ in the dark at RT. The subsequent steps of dehydration, infiltration, and embedding were the same as previously described (Li et al., 2016). The resin blocks were polymerized overnight at $60^{\circ} \mathrm{C}$ and sectioned with a diamond knife (Diatome) on a Leica Ultracut UCT 6 ultramicrotome (Leica Microsystems). Sections were poststained with $2 \%$ uranyl acetate in water and lead citrate. Images were acquired on a JEOL JEM $1200 \mathrm{EX}$ TEM equipped with a tungsten source at $120 \mathrm{kV}$ using a Morada SIS camera (Olympus). The electron-dense areas immediately 
A
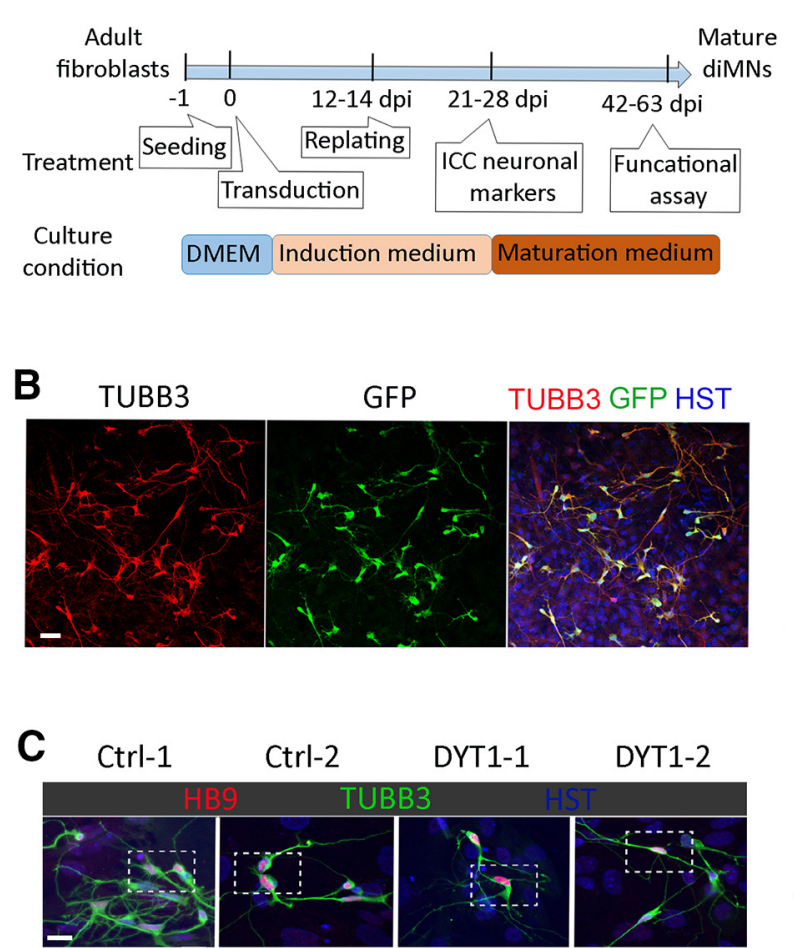

DYT1-2
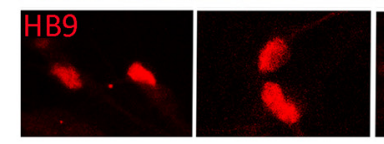

D $\div$

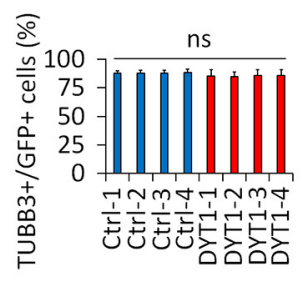

$\mathbf{F}$

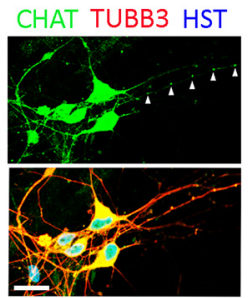

H

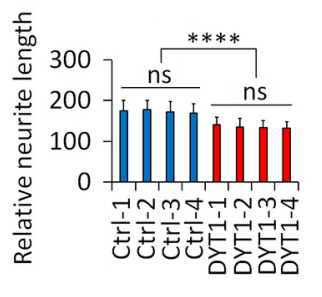

E

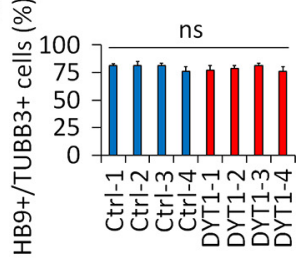

G

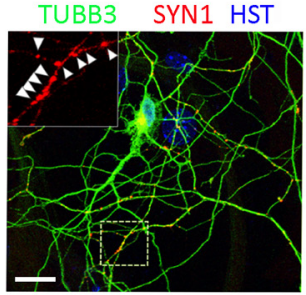

| $\overline{\bar{y}}$

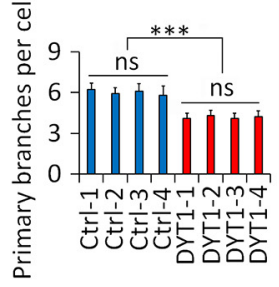

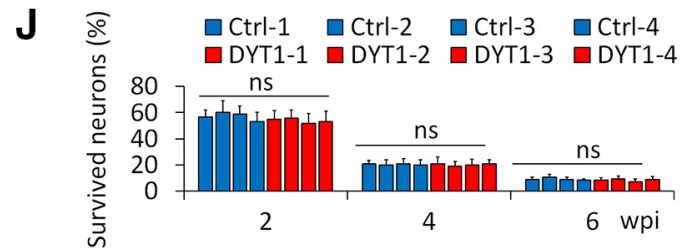

Figure 1. Direct conversion of DYT1 patient fibroblasts to MNs. $A, A$ schematic of the direct conversion procedure. $B$, A lower magnification view of the reprogrammed neurons at $19 \mathrm{~d}$ postviral infection (dpi). Virus-transduced cells are indicated by the co-expressed GFP. Scale bar: $50 \mu \mathrm{m}$. C, Confocal images of the reprogrammed neurons at $19 \mathrm{dpi}$. Higher magnification views of nuclear HB9 are shown in the bottom panels. Scale bar: $20 \mu \mathrm{m}$. D, Quantification of the reprogramming efficiency $(n=259$ for Ctrl-1, $n=249$ for Ctrl-2, $n=237$ for Ctrl-3, $n=243$ for Ctrl-4, $n=257$ for DYT1-1, $n=254$ for DYT1-2, $n=302$ for DYT1-3, and $n=257$ for DYT1-4 from triplicates; ns, not significant). $\boldsymbol{E}$, Fractions of diMNs among the reprogrammed neurons ( $n=257$ for Ctrl-1, $n=273$ for Ctrl-2, $n=252$ for Ctrl-3, $n=281$ for Ctrl-4, $n=229$ for DYT1-1, $n=219$ for DYT1-2, $n=289$ for DYT1-3, and $n=233$ for DYT1-4 from triplicates; ns, not significant). $\boldsymbol{F}$, Robust expression of the MN marker (hAT at 7 wpi. Arrowheads indicate ChAT + puncta. Scale bar: $20 \mu \mathrm{m}$. G, Expression of the presynaptic marker SYN1 at 9 wpi. The inset shows discrete SYN1+ puncta (indicated by arrow heads). Scale bar: $50 \mu \mathrm{m}$. $\boldsymbol{H}$, Quantitative analysis of neurite length at 2 wpi ( $n=123$ for Ctrl-1, $n=126$ for Ctrl-2, $n=137$ for Ctrl-3, $n=125$ for Ctrl-4, $n=114$ for DYT1-1, $n=127$ for DYT1-2, $n=118$ for DYT1-3, $n=113$ for DYT1-4 from triplicates; ns, not significant; $* * * * p=2.56 \mathrm{E}-05, t$ test). $I$, Neurite number of the primary branches examined at 4 wpi $(n=163$ for Ctrl-1, $n=153$ for Ctrl-2, $n=157$ for Ctrl-3, $n=145$ for Ctrl-4, $n=152$ for DYT1-1, and $n=143$ for DYT1-2, $n=125$ for DYT1-3, and $n=131$ for DYT1-4 from triplicates; ns, not significant; $* * * p=0.00032, t$ test). $J$, A time course analysis of diMN survival [each line $\mathrm{n}$ (neurons) $>2000$ from triplicates; ns, not significant].

beneath the nuclear membrane were measured with Image $(\mathrm{NIH})$ and the ratio of electron-dense area to the nuclear perimeter was used to evaluate the thickness of nuclear lamina. By using the ImageJ software, we zoomed in on the nuclear member and quantified the number of nuclear pore complexes (NPCs) and the pore sizes.

\section{Electrophysiology}

Neurons cultured on astrocyte-coated glass coverslips for $35 \mathrm{~d}$ or longer were used for analysis. Whole-cell patch-clamp recordings were performed under visual guidance using GFP-fluorescence to identify GFP+ cells when cells were maintained at $30^{\circ} \mathrm{C}$ in a submersion chamber with tyrode solution containing $150 \mathrm{~mm} \mathrm{NaCl}, 4 \mathrm{~mm} \mathrm{KCl}, 2 \mathrm{~mm} \mathrm{MgCl}_{2}, 3 \mathrm{~mm}$ $\mathrm{CaCl}_{2}, 10 \mathrm{~mm}$ glucose, and $10 \mathrm{~mm}$ HEPES at $\mathrm{pH} 7.4$ (adjusted with $\mathrm{KOH})$ and $300 \mathrm{mOsm}$. The recording pipettes $(\sim 6-9 \mathrm{M} \Omega)$ were filled with an intracellular solution containing $0.2 \mathrm{~mm}$ EGTA, $130 \mathrm{~mm}$ K-gluconate, $6 \mathrm{~mm} \mathrm{KCl}, 3 \mathrm{~mm} \mathrm{NaCl}, 10 \mathrm{~mm}$ HEPES, $4 \mathrm{~mm}$ ATP-Mg, $0.4 \mathrm{~mm}$ GTP-Na, $14 \mathrm{~mm}$ phosphocreatine-di(Tris) at pH 7.2 and $285 \mathrm{mOsm}$. Series and input resistance were measured in voltage-clamp with a $400 \mathrm{~ms}, 10-\mathrm{mV}$ step from a $-60-\mathrm{mV}$ holding potential (filtered at $30 \mathrm{kHz}$ and sampled at $50 \mathrm{kHz}$ ). Cells were used for analysis only if the series resistance was $<30 \mathrm{M} \Omega$ and was stable throughout the experiment. The input resistance ranged from 0.2 to $2 \mathrm{G} \Omega$. Currents were filtered at $3 \mathrm{kHz}$, acquired and digitized at $10 \mathrm{kHz}$ using Clampex 10.3 software (Molecular Devices). Action potentials (APs) were recorded in current-clamp and elicited by a series of current injections ranging from -20 to $200 \mathrm{pA}$ at $20-\mathrm{pA}$ increments and $800 \mathrm{~ms}$ in duration. All currentclamp recordings were made at resting membrane potential or without any current injection. Data analysis was performed with Clampfit 10.3 software (Molecular Devices). The AP trace immediately above threshold was used to determine the delay of first spike as the length of time and the start of current steps to the peak of AP. The same AP trace was used to measure AP threshold as the corresponding voltage when there was the sharpest change of the trace slope. The above indicated AP trace was also measured to determine maximum velocity of the rise and decay. AP frequency was obtained by dividing the maximum number of spikes during the current steps protocol with the step time duration $(800 \mathrm{~ms})$.

\section{Statistical analysis}

Unpaired two-tailed Student's $t$ tests were used to compare one experimental sample with its control. One-way analysis of variance was used when comparing multiple samples, with either Tukey's (when comparing samples with each other) or Dunnett's (when comparing samples to a control) post hoc tests. Results are expressed as mean \pm SD of at least three biological replicates, and $p<0.05$ is treated as significant. 
A

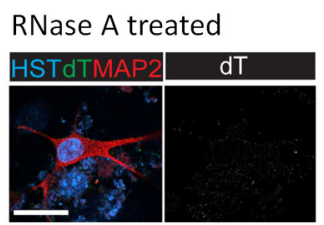

C

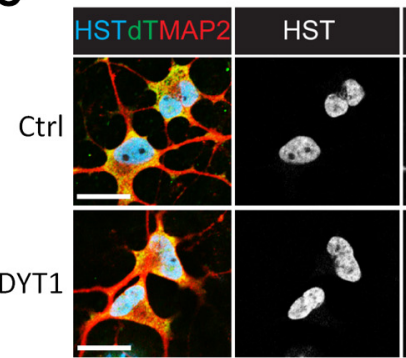

E

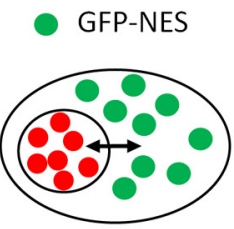

Normal NCT

F
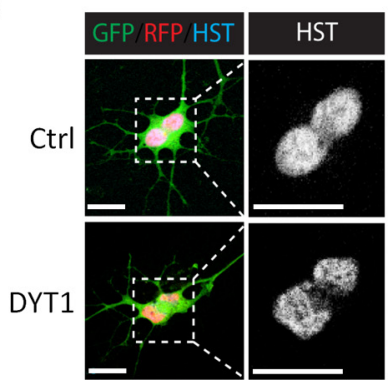

B Oligo-dA probe
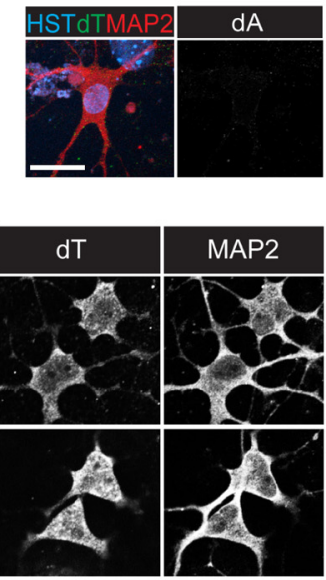

RFP-NLS

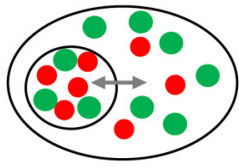

Impaired NCT

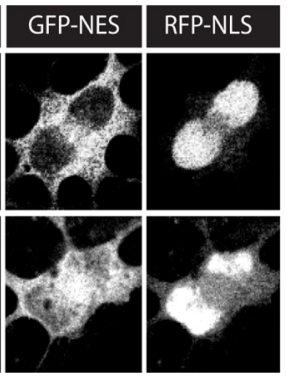

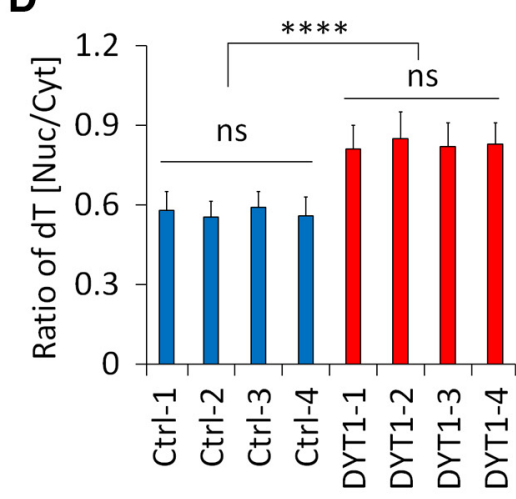

G
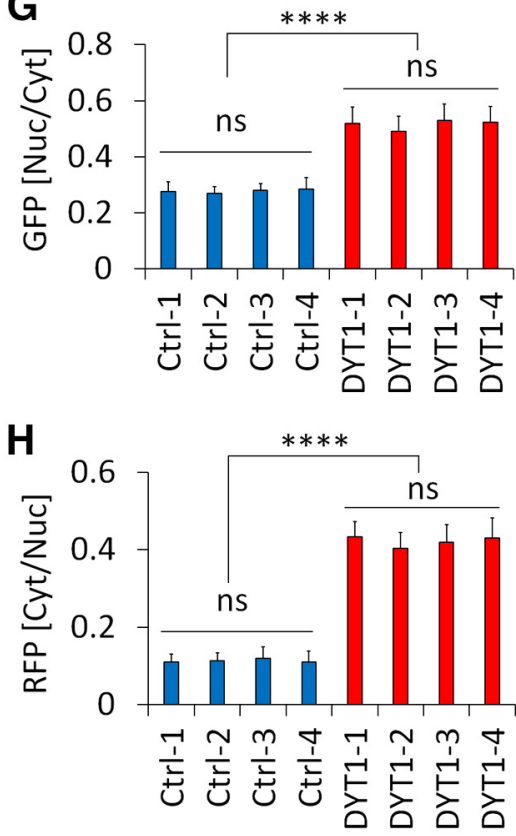

Figure 2. Impaired NCT in DYT1-diMNs from fibroblasts. $A$, RNase A-treated diMNs as negative controls for FISH. Cells were analyzed at 6 wpi. Scale bar: $20 \mu \mathrm{m}$. $\boldsymbol{B}$, 0 ligo-dA probes as negative controls for FISH assays at 6 wpi. Scale bar: $20 \mu \mathrm{m}$. C, FISH analysis of diMNs with oligo-dT probes at 6 wpi. Scale bar: $20 \mu \mathrm{m}$. D, Quantification of oligo-dT signal distribution $(n=65$ for Ctrl-1, $n=67$ for Ctrl-2, $n=72$ for Ctrl-3, $n=70$ for Ctrl-4, $n=64$ for DYT1-1, $n=63$ for DYT1-2, $n=74$ for DYT1-3, and $n=68$ for DYT1-4 from four replicates; ns, not significant; $* * * * p=3.83 \mathrm{E}-08, t$ test). $\boldsymbol{E}$, A dual reporter system for measuring protein NCT. NES, nuclear export signal; NLS, nuclear localization signal. $\boldsymbol{F}$, Representative confocal images of reporter distribution in diMNs at 6 wpi. Scale bar: $20 \mu \mathrm{m}$. G, Subcellular distribution of GFP-NES in diMNs ( $n=62$ for Ctrl-1, $n=64$ for Ctrl-2, $n=76$ for Ctrl-3, $n=74$ for Ctrl-4, $n=67$ for DYT1-1, and $n=68$ for DYT1-2 $n=75$ for DYT1-3, and $n=73$ for DYT1-4 from four replicates; ns, not significant; $* * * * p=5.15 E-10, t$ test). $H$, Subcellular distribution of RFP-NLS in diMNs ( $n=62$ for Ctrl-1, $n=64$ for Ctrl-2, $n=76$ for (trl-3, $n=74$ for Ctrl-4, $n=67$ for DYT1-1, and $n=68$ for DYT1-2 $n=75$ for DYT1-3, and $n=73$ for DYT1-4 from four replicates; ns, not significant; $* * * * p=4.15 \mathrm{E}-12, t$ test).

\section{Results}

Directly induced cholinergic MNs from fibroblasts of DYT1 patients

Because dystonia is a movement disorder and the ventral horn neurons in the spinal cord are most severely affected in Tor1a-mutant mice (Goodchild et al., 2005), we first generated MNs from human fibroblasts using a direct reprogramming method as we have previously described (Liu et al., 2016; Fig. 1A). The fibroblasts of four DYT1 patients and four age-matched healthy controls were used for this study. Although sex does not affect the reprogramming efficiency (Ding et al., 2020), all healthy controls were selected to be sex-matched to patient cell lines. Transduction with reprogramming factor-expressing lentivirus enabled adult human fibroblasts to gradually become neuron-like and grow complex neurites with time. Immunocytochemistry confirmed their expression of the neuronal marker TUBB3 (Fig. 1B) and early MN marker HB9
(Fig. 1C; Arber et al., 1999). Neuronal conversion efficiency was similar among all these fibroblasts, with $\sim 85 \%$ of virus-transduced GFP + cells as TUBB3+ neurons (Fig. 1D). Approximately $80 \%$ of these neurons showed strong HB9 expression and there was no difference between different cell lines (Fig. 1E). At later stages, such as seven or nineweeks postviral infection (wpi), reprogrammed neurons expressed high levels of ChAT and exhibited discrete puncta of the presynaptic marker synapsin 1 (SYN1; Fig. $1 F$, $G)$. Thus, fibroblasts from DYT1 patients as well as healthy controls could be efficiently and directly converted into mature cholinergic MNs, which were hereafter referred as diMNs (directly induced MNs).

\section{DYT1-diMNs show impaired development but normal} survival

DYT1 dystonia can also be classified as a neurodevelopmental disorder (Dauer, 2014). To track the development of human 
A
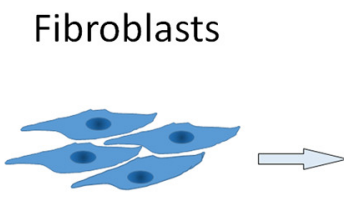

B

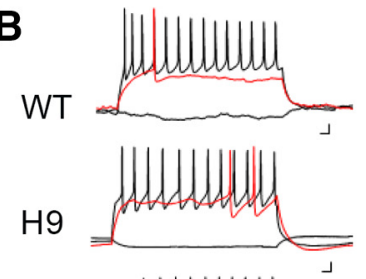

DYT1 \#11
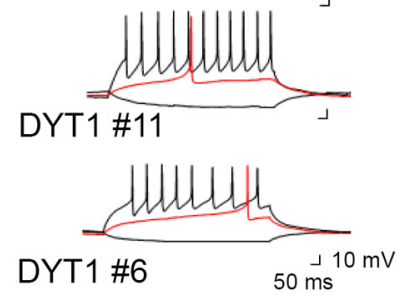

D

WT

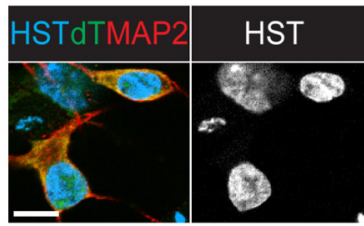

DYT1
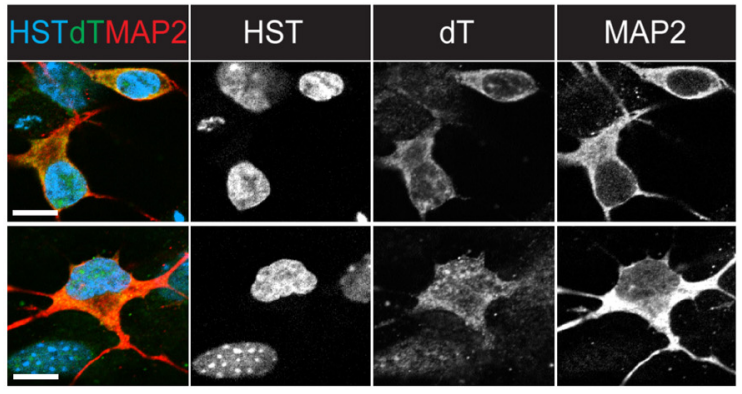

$\mathbf{F}$

WT
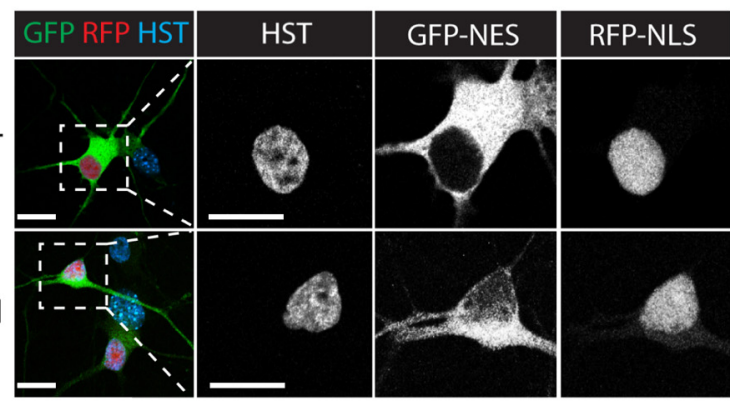

\section{iPSCs Neural progenitors iMNs}
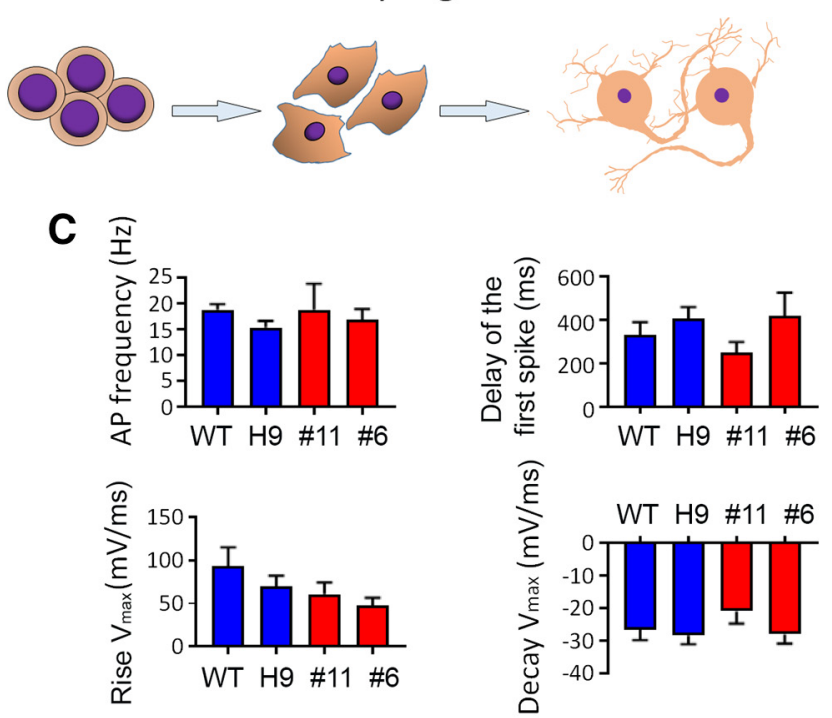

E

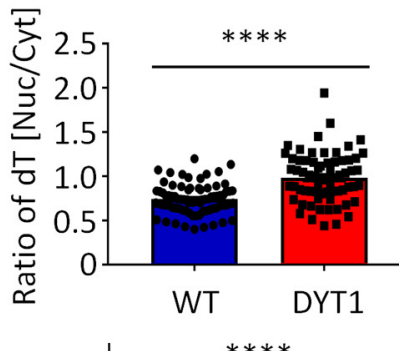

G

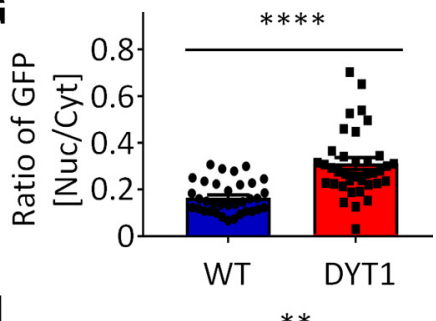

H

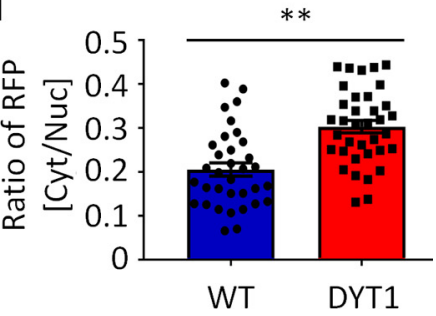

Figure 3. NCT impairments in DYT1-iMNs from iPSCs. $\boldsymbol{A}$, A schematic of the iPSC-based procedure to generate iMNs. $\boldsymbol{B}$, Repetitive AP waveforms recorded under current-clamp mode for the control (WT and H9) and two clones of DYT1 iMNs (\#11 and \#6) at 5 wpi. The precondition sweep and the sweep immediately above threshold (in red) are also shown. C, Quantifications of AP frequency, delay of the first spike, rise and delay $V_{\text {max }}$ in the indicated iMNs, respectively ( $n=30$ for each line from 4 biological replicates). $D$, Representative confocal images of FISH assay in iMNs at 3 wpi. Scale bar: $10 \mu \mathrm{m}$. $\boldsymbol{E}$, Subcellular distribution of mRNAs in iMNs by FISH assay ( $n=85$ for WT and 73 for DYT1 from triplicates; $* * * * p=7.13 \mathrm{E}-05, t$ test). $\boldsymbol{F}$, Representative confocal images of iMNs with the dual reporter at 3 wpi. Scale bar: $20 \mu \mathrm{m}$. G, Subcellular distribution of GFP-NES in iMNs $(n=34$ for WT and $n=36$ for DYT1 from triplicates; $* * * * p=3.42 E-06$, $t$ test). $\boldsymbol{H}$, Subcellular distribution of RFP-NLS in iMNs ( $n=34$ for WT and $n=36$ for DYT1 from triplicates; $* * p=0.0043, t$ test).

DYT1-diMNs, we examined neurite outgrowth and primary branches at the early reprogramming stages of 2 and 4 wpi as described in previous studies (Ding et al., 2013, 2016, 2018; Ding, 2015). DYT1-diMNs had significantly shorter neurites (Fig. $1 H$ ) and fewer primary branches (Fig. 1I) than controls. On the other hand, no significant differences were identified on neuronal survival within six weeks (Fig. 1J). These results support the notion that DYT1 dystonia is caused by alterations in neuronal function rather than by neurodegeneration (Granata et al., 2009).
DYT1-diMNs exhibit dysfunctional nucleocytoplasmic transport (NCT)

Torsin has been shown to participate in nuclear export of mRNAs via a non-conventional mechanism called NE budding in Drosophila (Speese et al., 2012). In Torsin mutants, disruption of mRNA nuclear export led to slowed growth and impaired development and plasticity of neuromuscular junctions (Jokhi et al., 2013). To examine whether mRNA export was similarly impaired in DYT1-diMNs, we performed FISH, as previously described (Packard et al., 2015; Li et al., 2016; Ding et al., 2020). 

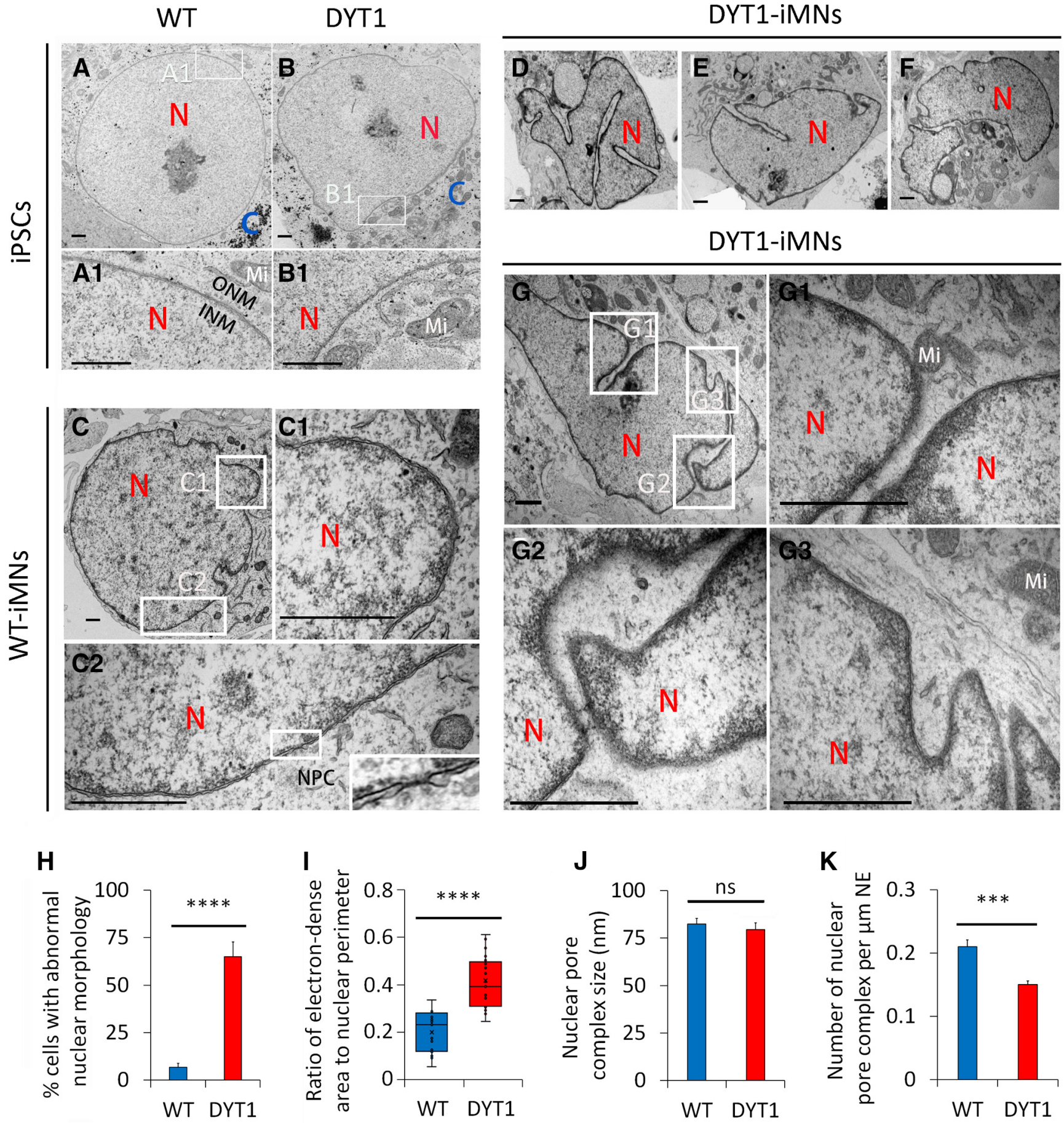

Figure 4. NE deformation in DYT1-iMNs. $\boldsymbol{A}$, Representative electron micrograph of WT-iPSC. A higher magnification view of the boxed region is shown in $\boldsymbol{A} \mathbf{1}$ of the bottom panel. N, nucleus; C, cytoplasm; INM, inner nuclear membrane; ONM, outer nuclear membrane; Mi, mitochondria. Scale bars: $1 \mu \mathrm{m}$. $\boldsymbol{B}$, Representative electron micrograph of DYT1-iPSC. A higher magnification view of the boxed region is shown in $\boldsymbol{B} 1$ of the bottom panel. Scale bars: $1 \mu \mathrm{m}$. $\boldsymbol{C}$, Representative electron micrographs of WT-iMN at 3 wpi. Higher magnification views of the boxed regions are shown in $\mathbf{C 1}, \mathbf{C 2}$, respectively. NPC, nuclear pore complex. Scale bars: $1 \mu \mathrm{m}$. D-G, Representative electron micrographs of DYT1-iMNs at 3 wpi. Higher magnification views of the boxed regions are shown in $\mathbf{G 1}, \mathbf{G}$, G3, respectively. Scale bars: $1 \mu \mathrm{m}$. $\boldsymbol{H}$, iMNs with abnormal nuclear morphology at 3 wpi $[n$ (nuclei) $=47$ for WT and 42 for DYT1 from triplicates; $* * * * p=4.31 \mathrm{E}-05, t$ test]. $I$, Ratio of electron dense area immediately beneath the nuclear membrane to the nuclear perimeter in iMNs at 3 wpi $[n$ (nuclei) $=24$ for WT and 22 for DYT1 from triplicates; $* * * * p=2.15 \mathrm{E}-05, t$ test]. J, Nuclear pore size in iMNs at 3 wpi [ $n$ (nuclei/nuclear pore) $=17 / 56$ for WT and 21/34 for DYT1 from triplicates; ns, not significant, $t$ test]. $\boldsymbol{K}$, Number of nuclear pore structure in iMNs at 3 wpi $[n$ (nuclei) $=35$ for WT and 32 for DYT1 from triplicates; $* * * p=0.000263, t$ test].

We used DIG-labeled oligo-dT as probes to detect the subcellular distribution of Poly (A) mRNAs. FISH specificity was confirmed by a lack of signals when samples were treated with RNase A or when DIG-labeled oligo-dA was used as a probe (Fig. $2 A, B$ ). mRNA nuclear export was measured by the ratio of nuclear to cytoplasmic oligo-dT signals $\left(\mathrm{dT}_{\text {nud }} / \mathrm{dT}_{\text {cyt }}\right)$. In healthy control diMNs, most mRNAs were localized in the cytoplasm when examined at 6 wpi (Fig. 2C,D). In contrast, substantial amounts of mRNAs were accumulated inside the nucleus of DYT1diMNs (Fig. 2C,D). 
A
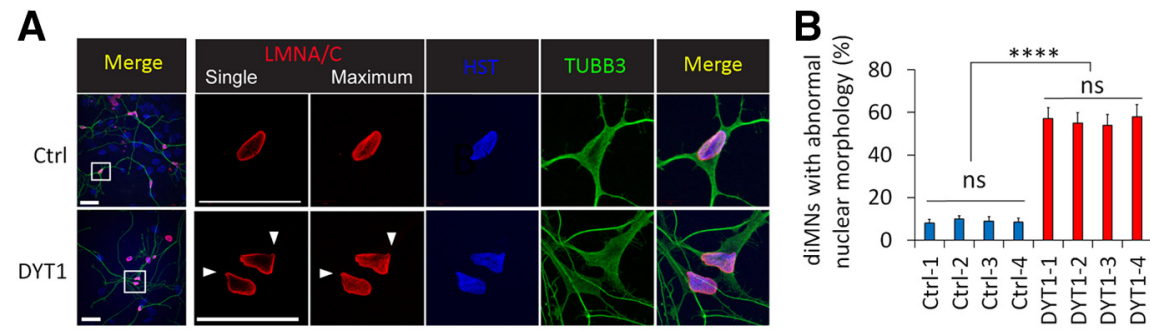

c
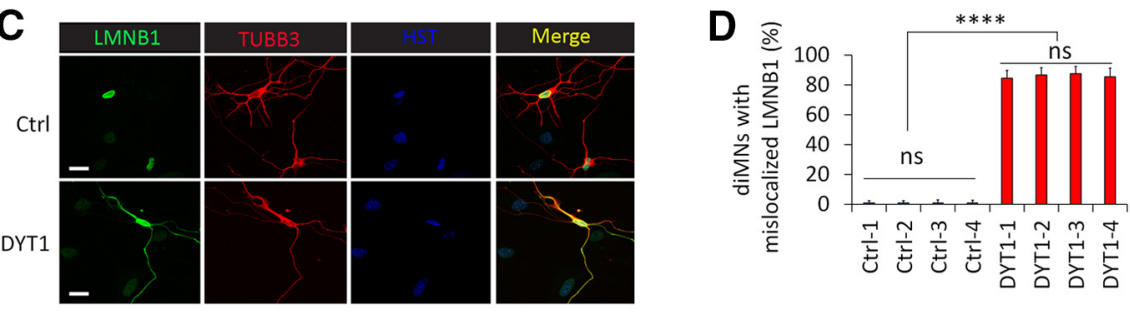

E

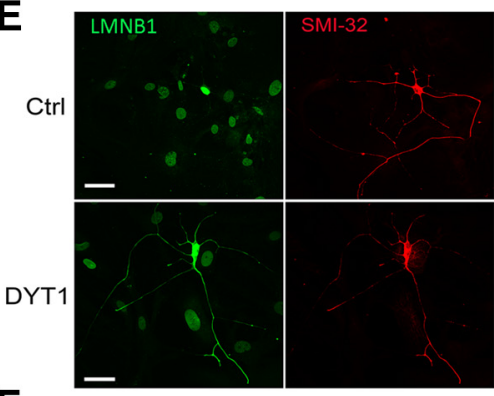

$\mathbf{F}$
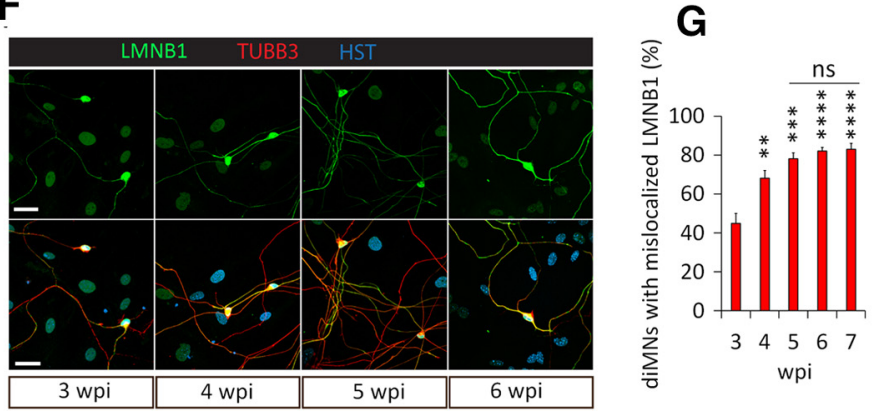

Figure 5. Abnormal nuclear morphology and mislocalized LMNB1 in DYT1-diMNs. $\boldsymbol{A}$, Representative confocal images of diMNs at 4 wpi. Arrowheads indicate the sharp and rigid angles in nuclear membrane. Scale bar: $50 \mu \mathrm{m}$. $\boldsymbol{B}$, Quantification of diMNs with abnormal nuclear morphology at $4 \mathrm{wpi}(n>100$ for each line from four replicates; ns, not significant; $* * * * p=2.38 \mathrm{E}-09$, $t$ test). $\boldsymbol{C}$, Representative confocal images of diMNs at 6 wpi. Scale bar: $20 \mu \mathrm{m}$. D, Percentages of diMNs with mislocalized LMNB1 at 6 wpi ( $n=410$ for Ctrl-1, $n=418$ for Ctrl-2, $n=490$ for Ctrl-3, $n=378$ for Ctrl-4, $n=358$ for DYT1-1, $n=349$ for DYT1-2, $\mathrm{n}=378$ for DYT1-3, and $\mathrm{n}=376$ for DYT1-4 from four replicates; $\mathrm{ns}$, not significant; $* * * * p=6.43 \mathrm{E}-11, t$ test). $\boldsymbol{E}$, Representative confocal images of diMNs at 6 wpi. Scale bar: $50 \mu \mathrm{m}$. $\boldsymbol{F}$, A time course analysis of LMNB1 mislocalization in DYT1diMNs. Scale bar: $50 \mu \mathrm{m}$. G, Frequency of LMNB1 mislocalization in DYT1-diMNs at the indicated time points $(n=458$ for 3 wpi, $n=425$ for 4 wpi, $n=327$ for 5 wpi, $n=314$ for 6 wpi, and $n=213$ for 7 wpi from triplicates; ns, not significant; compare to 3 wpi, $* * p<0.01, * * * p<0.001, * * * * p<0.0001$, ANOVA). $\boldsymbol{H}$, Subcellular distribution of LMNB1 (neurite vs nucleus) in DYT1-diMNs at the indicated time points ( $n=126$ for 3 wpi, $n=114$ for 4 wpi, $n=128$ for 5 wpi, and $n=107$ for 6 wpi from triplicates; ns, not significant; $* * * p<0.001, * * * * p<0.0001$, ANOVA or $t$ test).

Interestingly, we noticed obvious MAP2 signals in the nucleus of DYT1-diMNs (Fig. 2C), suggesting that protein NCT might also be compromised. We used a dual reporter system (2Gi2R) to further examine NCT (Ding et al., 2020). This reporter system consists of a fused double-GFP with a nuclear export signal (2GFP-NES) and a double RFP containing a nuclear localization signal (2RFP-NLS; Mertens et al., 2015). In cells with normal NCT activity, GFP and RFP are localized in the cytoplasm and nucleus, respectively; in cells with compromised NCT, this subcellular distribution will be disrupted (Fig. 2E). An increased ratio of nuclear to cytoplasmic GFP ( $\left.\mathrm{GFP}_{\text {nuc }} / \mathrm{GFP}_{\text {cyt }}\right)$ represents

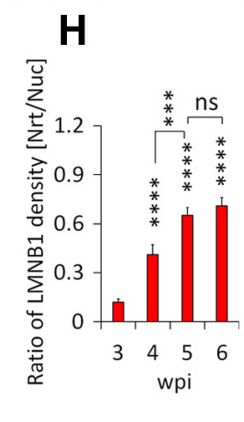

impaired protein export, while a higher ratio of cytoplasmic to nuclear RFP ( $\left.\mathrm{RFP}_{\text {cyt }} / \mathrm{RFP}_{\text {nuc }}\right)$ indicates compromised protein nuclear import. Both GFP and RFP were mainly localized in the cytoplasm and nucleus, respectively, in control diMNs when examined at 6 wpi (Fig. $2 F-H$ ). In contrast, DYT1-diMNs had a substantial amount of GFP accumulated inside the nucleus and obvious RFP detected in the cytoplasm (Fig. 2F). As such, the ratios of $\mathrm{GFP}_{\text {nud }} / \mathrm{GFP}_{\text {cyt }}$ and $\mathrm{RFP}_{\text {cyt }} / \mathrm{RFP}_{\text {nuc }}$ were significantly higher for DYT1diMNs than control neurons (Fig. $2 G, H)$. Together, FISH analysis and dual reporter assays clearly indicate that both mRNA nuclear export and protein NCT are impaired in DYT1diMNs.

\section{NCT impairments in MNs from DYT1 iPSCs}

To verify our findings, we examined iPSC-derived MNs (Fig. 3A). One iPSC clone from a healthy control (WT) and two clones from a DYT1 patient (\#6 and \#11) were successfully generated. The well-characterized human ESC line H9 was included as an additional control. We differentiated these pluripotent stem cells into neural progenitors (Yang et al., 2014), which were further induced to become MNs (Tang et al., 2017; Sepehrimanesh and Ding, 2020). These iPSCs-derived or ESCs-derived MNs were hereafter referred as iMNs. Electrophysiological properties of iMNs were examined by whole-cell patchclamp recordings at 4 wpi. All patched iMNs fired repetitive APs on current injections (Fig. 3B). No apparent differences were observed between DYT1 and control iMNs on AP frequency, delay of the first spike, and rise and decay of $\mathrm{V}_{\max }$ (Fig. 3C). Interestingly, nuclear mRNA export and protein NCT were significantly compromised in DYT1iMNs (Fig. 3D-H), though these defects were less severe than in DYT1-diMNs (Fig. 2). For example, the cytoplasmic localization of RFP-NLS was less obvious in DYT1-iMNs than DYT1-diMNs (compare Figs. $3 F, H$ and $2 F, H$ ). Such a difference may reflect an aging effect on disease phenotypes, since diMNs retain aging-associated features, whereas iMNs are rejuvenated during the reprogramming process (Mertens et al., 2015; Tang et al., 2017).

\section{Abnormal NE but no perinuclear blebs in DYT1 MNs}

One cellular hallmark in Torla-mutant mice (Torla knock-out or Tor $1 a^{\Delta E / \Delta E}$ ) is the abnormal NE morphology in multiple areas of the CNS (Goodchild et al., 2005; Tanabe et al., 2016). It is characterized by an accumulation of blebs in the perinuclear 
A

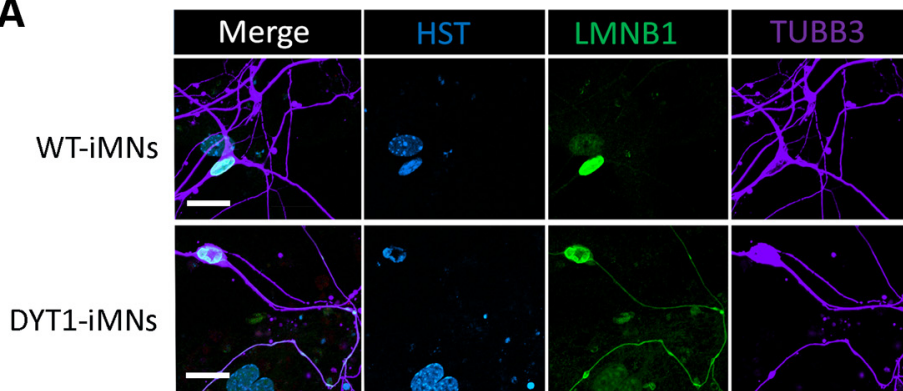

C
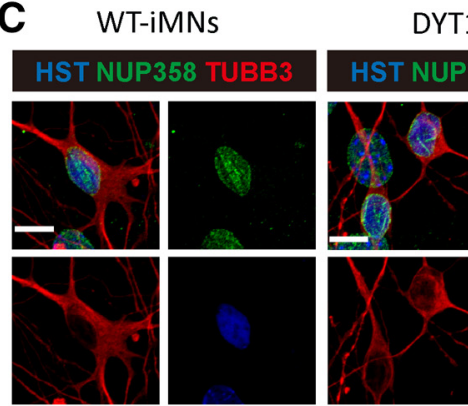

DYT1-iMNs

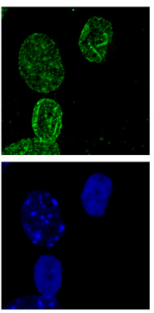

E

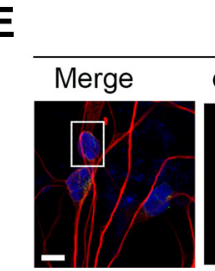

WT-iMNs
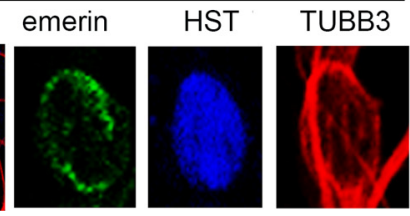

Figure 6. LMNB1-specific mislocalization in DYT1-iMNs. A, Representative confocal images of the indicated markers in iMNs at 3 wpi. Scale bar: $20 \mu \mathrm{m}$. $\boldsymbol{B}$, Subcellular localization of LMNB1 in iMNs at 3 wpi ( $n=397$ for WT and $n=423$ for DYT1 from triplicates; $* * * * p=2.42 E-06, t$ test). C $E$, Representative confocal images of the indicated markers in iMNs at 3 wpi. Scale bars: $10 \mu \mathrm{m}$.

space, morphologically similar to the budding granules in the body-wall muscle in torsin-mutant flies (Speese et al., 2012; Jokhi et al., 2013). We first examined whether such abnormalities also occur and contribute to impaired NCT in human DYT1 neurons. Nuclear morphology was studied by TEM. At the iPSC stage, both WT and DYT1 cells had smooth and round nuclei with clear double nuclear membranes, except for a few small wrinkles in some DYT1-iPSCs (Fig. 4A,B). After differentiation into mature iMNs, most WT cells retained the regular, smooth nuclear shapes with occasional wrinkles and light invaginations (Fig. 4C). In contrast, the nuclear morphology of DYT1-iMNs showed many sharp angles and deep invaginations (Fig. 4D-H). However, we failed to observe any bleb structures at the perinuclear space in DYT1-iMNs from three batches of samples. If bleb structures exist, the frequency must be very low. Such a result indicates that the mechanisms underlying the abnormal NCT might differ between human DYT1 patients and other animal models such as Drosophila (Jokhi et al., 2013) and mouse (Goodchild et al., 2005; Tanabe et al., 2016). Interestingly, we found that DYT1-iMNs exhibited thickened nuclear lamina with high electron-dense areas immediately beneath the nuclear membranes, especially in regions with abnormal angles and invaginations (Fig. 4G1-G3). Quantification showed a much higher ratio of the electron-dense area to the nuclear perimeter in DYT1-iMNs than controls (Fig. 4I). Interestingly, although the NPC size was comparable between WT-iMNs and DYT1-iMNs (Fig. 4J), the number of NPCs at TEM was significantly lower in DYT1 neurons (Fig. 4K). These results agree with a recent report showing that Torsin functions in nuclear pore biogenesis
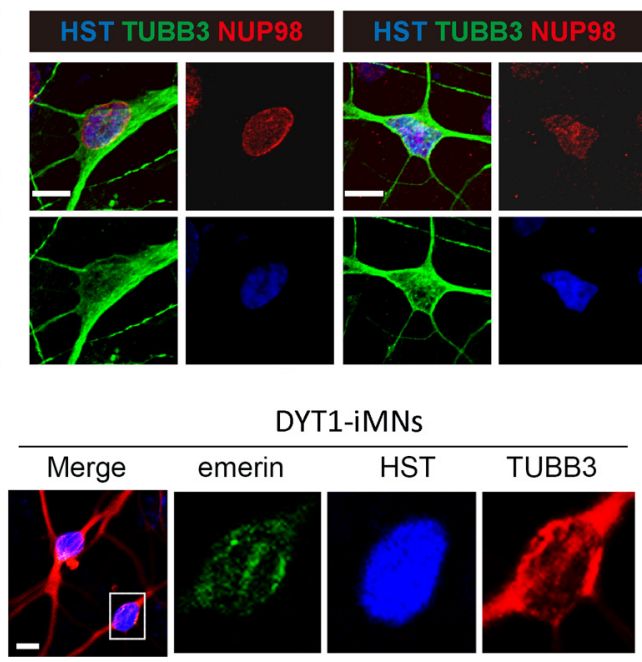

B

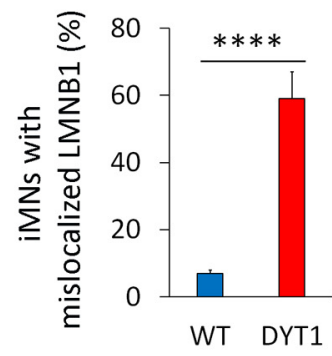

DYT1-iMNs

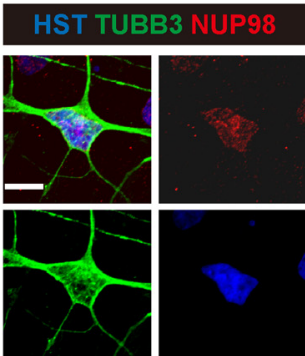

DYT1-iMNs
(Rampello et al., 2020) and suggest that the impaired NCT in DYT1 neurons might be because of a reduction of NPC number.

\section{LMNB1 mislocalization in DYT1 MNs}

Since deformed NE and thickened nuclear lamina are salient features of DYT1-iMNs (Fig. 4G,I), they were further examined by immunocytochemistry and confocal microscopy. Nuclear lamina consists of A-type and B-type lamins, which are intermediate filament proteins and form the meshwork beneath the inner nuclear membrane to support the nuclear morphology and structural integrity of the NE (Capell and Collins, 2006; Burke and Stewart, 2013; Shah et al., 2017; Tatli and Medalia, 2018). LMNA and LMNC are the major A-type lamins encoded by a single LMNA gene in humans (Burke and Stewart, 2013). Staining for LMNA/C showed their exclusive localization in the nuclear periphery in both control and DYT1-diMNs (Fig. 5A). However, unlike the regular ovoid nucleus with a smooth outline in control cells, $\sim 60 \%$ of DYT1-diMNs showed abnormal nuclear morphology with sharper and rigid angles (Fig. 5A,B), consistent with what was observed under TEM (Fig. 4D-H).

Similar to LMNA/C, staining of the major B-type nuclear lamins LMNB1 revealed its normal nuclear localization in control diMNs (Fig. 5C). Unexpectedly, a substantial amount of LMNB1 were detected in the cytoplasm and neurites of DYT1diMNs when examined at 6 wpi (Fig. 5C). Quantification showed $\sim 80 \%$ of DYT1-diMNs exhibited mislocalized LMNB1, whereas such mislocalization was rarely detected in healthy controls (Fig. 5D). Co-staining with SMI-32 and MAP2 indicated that the mislocalized LMNB1 was distributed in both axons and dendrites 
A

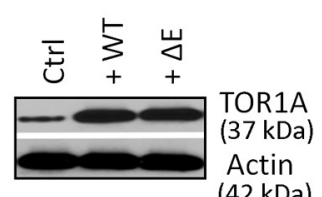

B

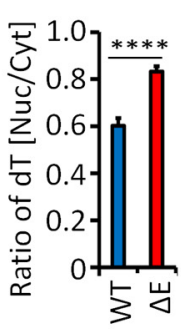

C

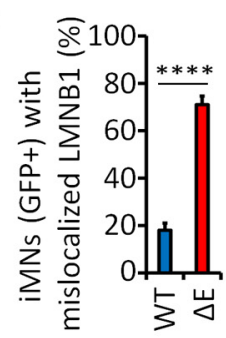

D

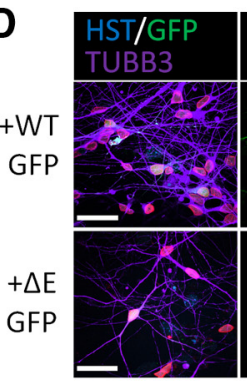

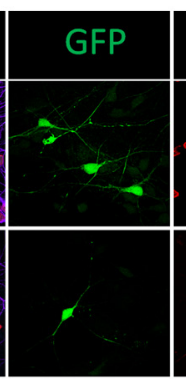

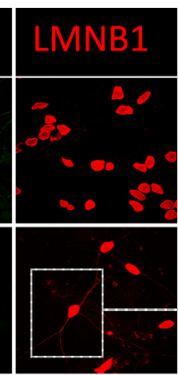

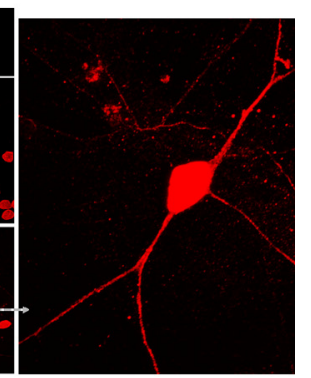

E

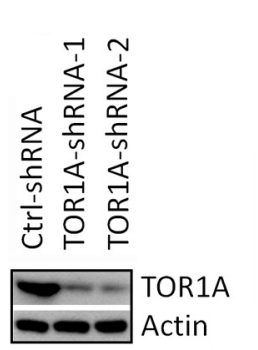

$\mathbf{F}$

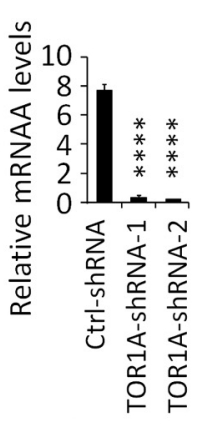

G

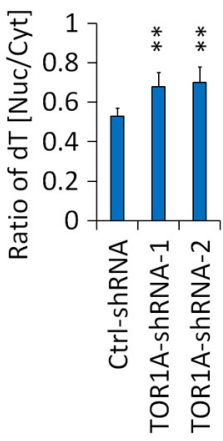

H

Ctrl-
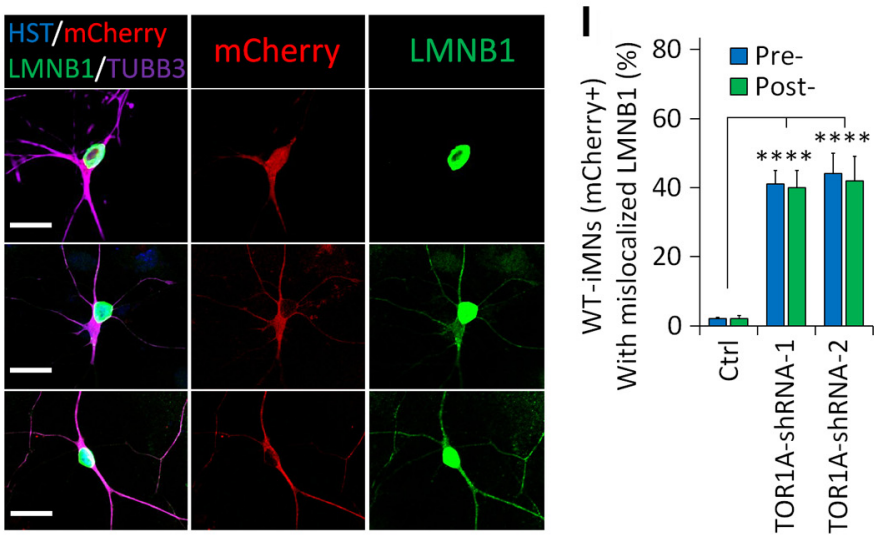
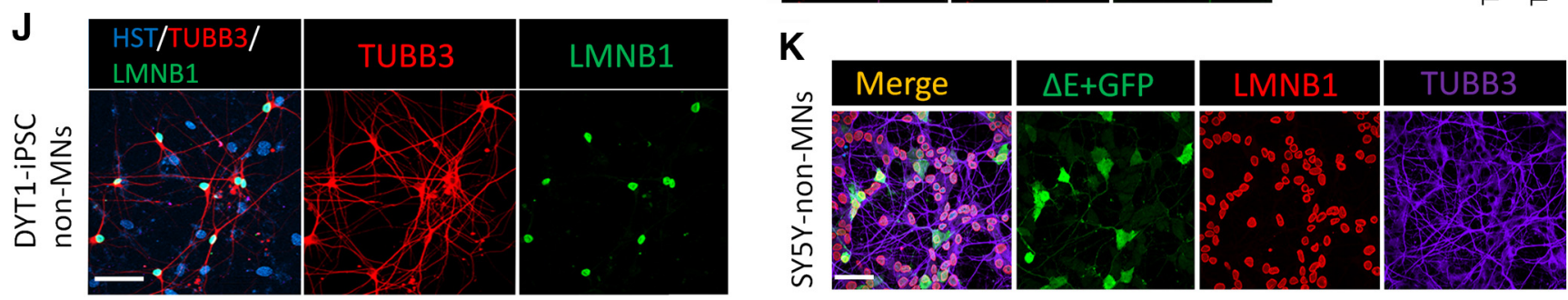

Figure 7. TOR1A hypoactivity disrupts NCT and LMNB1 localization in a MN-specific manner. A, Protein expression of the ectopically expressed TOR1A WT or $\triangle E$ in iMNs; Actin serves as a loading control. $\boldsymbol{B}$, The effect of TOR1A expression on mRNA distribution assayed by FISH in iMNs at 3 wpi ( $n=99$ for WT and $n=78$ for DYT1 from triplicates; ***** $=5.44 \mathrm{E}-05, t$ test). $\boldsymbol{C}$, Quantification of LMNB1 mislocalization in iMNs at 3 wpi ( $n=82$ for WT and $n=75$ for DYT1 from triplicates; $* * * * p=2.48 \mathrm{E}-06, t$ test). D, Representative confocal images of iMNs with ectopic (GFP+) TOR1A WT or $\Delta \mathrm{E}$ at 3 wpi. Scale bar: $50 \mu \mathrm{m}$. $\boldsymbol{E}$, Western blots showing shRNA knock-down of TOR1A protein expression in iMNs. $\boldsymbol{F}$, qRT-PCR showing downregulation of TOR1A mRNA by shRNAs $(n=3 ; * * * * p=6.58 \mathrm{E}-05$, ANOVA). $G$, The effect of TOR1A downregulation on mRNA subcellular distribution assayed by FISH in iMNs at 3 wpi ( $n=95$ for Ctrl, $n=87$ for TOR1A-shRNA-1, and $n=78$ for TOR1A-shRNA-2 from triplicates; $* * p=0.00327$, ANOVA). $\boldsymbol{H}$, Representative confocal images of iMNs with the indicated shRNAs (mCherry + ) at 3 wpi. Scale bar: $25 \mu \mathrm{m}$. $I$, The effect of TOR1A downregulation on LMNB1 subcellular distribution in iMNs. ShRNA lentiviruses were added at either predifferentiation (neural progenitors) or postdifferentiation (MNs at 5 dpi) stages; $n$ (pre and post) $=126$ and 113 for Ctrl, $n=118$ and 97 for TORTA-shRNA-1, and $n=103$ and 94 for TOR1A-shRNA-2 from triplicates; $* * * * p=4.08 \mathrm{E}-10$ (Ctrl vs shRNA-1) and 5.78E-9 (Ctrl vs shRNA-2), $t$ test. J, Representative confocal images of DYT1-iPSC spontaneously differentiated non-MNs at 3 wpi. Scale bar: $100 \mu \mathrm{m}$. $\boldsymbol{K}$, Representative confocal images of SY5Y-derived non-MNs with ectopic expression of TOR1A $\triangle E$ (GFP+) at 4 wpi. Scale bar: $50 \mu \mathrm{m}$.

(Fig. 5E). A time course analysis revealed that cytoplasmic LMNB1 in DYT1-diMNs became progressively more frequent, with $45 \%$ at 3 wpi and $80 \%$ at $6-7$ wpi (Fig. $5 F, G)$. The cytoplasmic fraction of LMNB1 also greatly increased with time and reached a plateau at 6 wpi (Fig. $5 H$ ). Importantly, LMNB1 mislocalization was also observed in DYT1-iMNs from iPSCs (Fig. $6 A$ ). Such mislocalization could be detected as early as $1.5 \mathrm{wpi}$ and in $\sim 60 \%$ of DYT1-iMNs when examined from 2 to 8 wpi (Fig. $6 B$ ). In contrast, we failed to detect any obvious mislocalization of several other nuclear proteins, including LMNA/C (Fig. $5 A$ ), the nuclear pore subunits NUP98 (Fig. 6C) and NUP358 (Fig. $6 D$ ), and the inner nuclear membrane protein emerin (Fig. $6 E$ ) in DYT1-iMNs.

TOR1A hypoactivity disrupts NCT and LMNB1 localization To determine whether these above defects were caused by dysregulation of TOR1A or largely influenced by the genetic background of human patients, we employed two approaches. One was to ectopically express either TOR1A or TOR1A $\Delta \mathrm{E}$ mutant in WT-iMNs. These MNs could be identified by the co-expressed GFP reporter. Western blot analysis showed that both TOR1A and TOR1A $\Delta \mathrm{E}$ were expressed at a similar level (Fig. $7 A$ ). Interestingly, ectopic TOR1A $\triangle \mathrm{E}$ mutant but not the WT TOR1A disrupted mRNA nuclear export (Fig. $7 B$ ) and LMNB1 subcellular distribution (Fig. $7 C, D$ ). In a second approach, we downregulated TOR1A expression through shRNAs in WT-iMNs. shRNA-expressing cells were marked by the co-expressed mCherry reporter. Each of the two separate shRNAs against TOR1A could efficiently down-regulate its expression at both protein and mRNA levels (Fig. $7 E, F$ ). Consistently, downregulation of endogenous TOR1A led to defective export of nuclear mRNAs (Fig. 7G) and altered subcellular localization of LMNB1 (Fig. 7H). Such LMNB1 mislocalization was independent of the neuronal differentiation process because MNs infected with the TOR1A-shRNA virus at either predifferentiation (neuronal progenitors) or postdifferentiation (MNs at $5 \mathrm{dpi}$ ) stages exhibited similar results (Fig. 7I). Together, these results strongly support that NCT impairment and LMNB1 mislocalization are caused by TOR1A hypoactivity. 

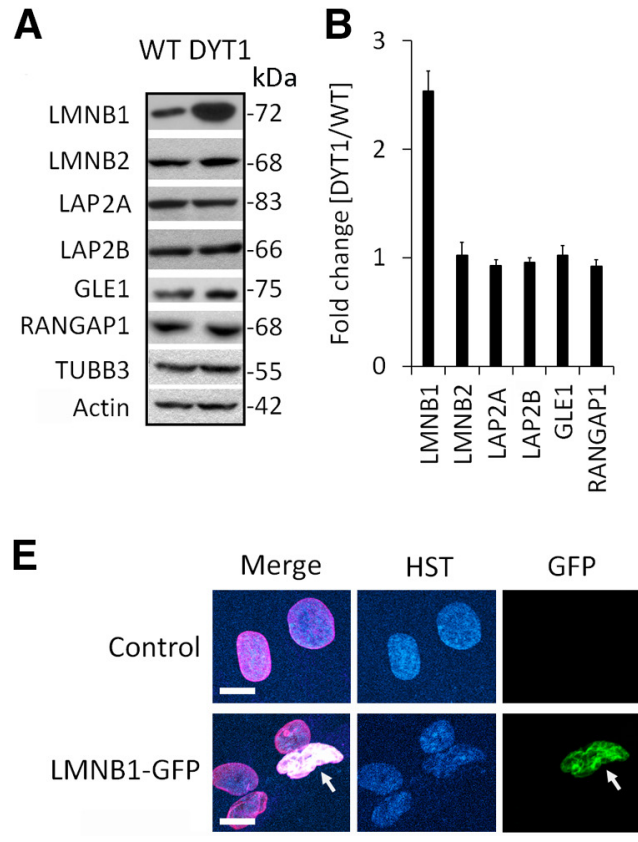

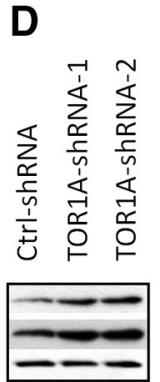

LMNB1 (72 kDa) Longer exposure Actin (42 kDa)

1.01 .82 .1 Relative levels

Figure 8. Specific upregulation of LMNB1 in DYT1 MNs. $A$, Western blottings for the indicated proteins in WT-iMNs and DYT1-iMNs at 11 dpi. TUBB3 and Actin are loading controls. $B$, Fold changes (DYT1 to WT) of the indicated proteins in $\boldsymbol{A}$ after normalization to the loading control $(n=3)$. C, Relative LMNB1 mRNA levels analyzed by qRT-PCR in iMNs at 11 dpi $(n=3$; $* * p=0.0092, t$ test). $\boldsymbol{D}$, Western blottings for LMNB1 expression (shorter and longer exposure) in iMNs with the indicated shRNAs at 11 wpi. Actin serves as a loading control. Values at the bottom indicate the relative LMNB1 protein levels. $\boldsymbol{E}$, Confocal micrographs of fibroblasts with or without overexpression of LMNB1 (fused with GFP). Arrow indicates a cell with LMNB1 overexpression. Scale bars: $10 \mu \mathrm{m}$.

To examine whether hypoactivity of TOR1A also leads to LMNB1 mislocalization in other neuronal subtypes, we generated non-MNs via two approaches. One was spontaneous differentiation of DYT1 neural progenitors into generic neurons (Fig. 7J), which mainly consisted of glutamatergic and GABAergic neurons (Sepehrimanesh and Ding, 2020). The second approach was to ectopically express TOR1A $\triangle E$ in generic neurons that were differentiated from human SH-SY5Y neuroblasts (Fig. $7 \mathrm{~K}$ ). Interestingly, we did not notice any mislocalized LMNB1 in either of these generic neurons (Fig. 7J,K). These results suggest that TOR1A hypoactivity may cause LMNB1 mislocalization in a MN-specific manner.

\section{Specific upregulation of LMNB1 in DYT1 MNs}

LMNB1 expression in iPSCs-derived iMNs was then examined by western blot analysis. Beta-actin and the neuron-specific microtubule TUBB3 were used as protein loading controls. Strikingly, the LMNB1 protein level was dramatically higher (2.5-fold) in DYT1-iMNs than in WT controls (Fig. 8A,B). Such a result was consistent with immunocytochemistry showing brighter LMNB1 signals and cytoplasmic accumulation in DYT1-diMNs (Fig. 5C,E). Notably, qRT-PCR analysis showed that $L M N B 1$ transcripts in DYT1-iMNs were 3- to 5-fold higher than those in WT controls (Fig. 8C), indicating that increased LMNB1 protein was mainly contributed by upregulated gene expression. In sharp contrast, no significant differences were observed for lamin-associated proteins, LAP2A and LAP2B (Fig. $8 A, B)$, which are known to interact with LMNA/C and LMNB1 (Wilson and Foisner, 2010). We also failed to detect any significant changes on LMNB2 (another B-type nuclear lamina), GLE1 (a factor involved in mRNA export), and RANGAP1 (a nuclear pore-associated protein involving in nuclear transport; Fig. $8 \mathrm{~A}$, $B)$. LMNB1 protein levels were also increased in WT-iMNs when TOR1A was downregulated by shRNAs (Fig. $8 D$ ). These results indicate that LMNB1 is uniquely dysregulated on both protein expression and subcellular localization in DYT1 MNs or MNs with hypoactive TOR1A. To directly examine the effect of LMNB1 upregulation, we tried several batches of experiments to overexpress LMNB1 in MNs. Nonetheless, all MNs with ectopic LMNB1 (indicated by the co-expressed GFP) died soon after virus infection. As an alternative, we examined LMNB1 overexpression in fibroblasts of a healthy human patient. Interestingly, cells with ectopic LMNB1 $(\mathrm{GFP}+)$ showed a severely deformed nuclear morphology (Fig. 8E), supporting the hypothesis that LMNB1 upregulation underlies the abnormal nuclear morphology in DYT1-MNs.

\section{Phenotypic amelioration of DYT1 MNs via LMNB1 downregulation}

To determine whether LMNB1 dysregulation contributes to the pathologic phenotypes in DYT1 MNs, we knocked down its expression through a shRNA approach. The shRNA-expressing cells were marked with the co-expressed mCherry reporter. A shRNA against luciferase was used as a control. Two shRNAs were identified to be efficient in reducing the expression of endogenous LMNB1 determined by both western blots and immunocytochemistry (Fig. 9A,B). Interestingly, downregulation of LMNB1 resulted in a much-reduced frequency of LMNB1 mislocalization in DYT1-iMNs (Fig. 9C). It also significantly improved neurite outgrowth (Fig. $9 D$ ), increased the number of primary branches (Fig. $9 E$ ), and markedly reduced the percentage of cells with abnormal nuclear morphology (Fig. 9F). When compared with the control shRNA, FISH analysis with DIG-labeled oligo-dT probes showed that LMNB1-shRNAs significantly reduced nuclear accumulation of mRNAs in DYT1-iMNs (Fig. 9G,H). Together, these results clearly indicate that LMNB1 dysregulation is a salient pathologic feature contributing to the delayed neuronal development, abnormal nuclear morphology, and impaired NCT. 

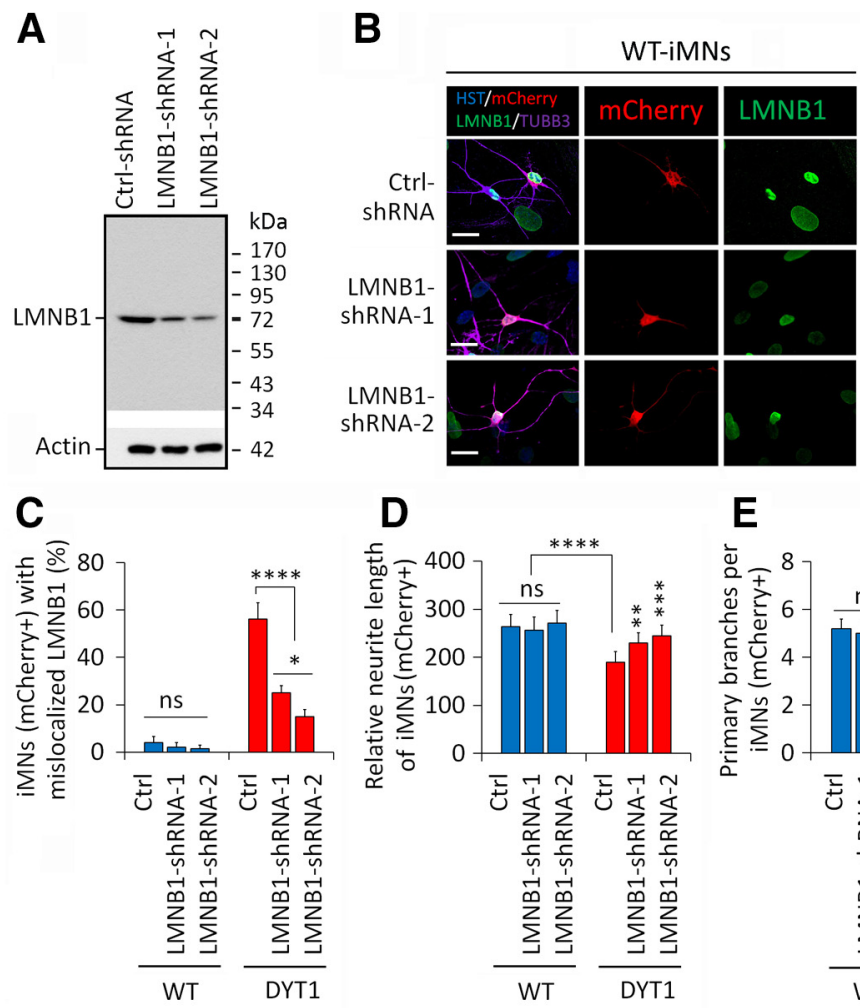

\section{E}

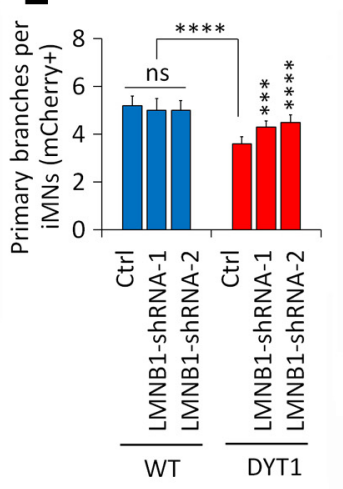

$\mathbf{F}$

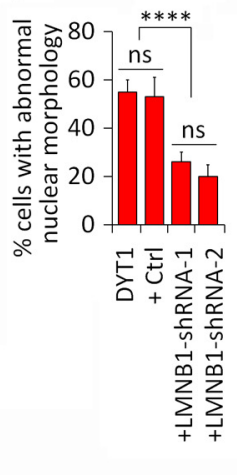

G

WT-iMNs
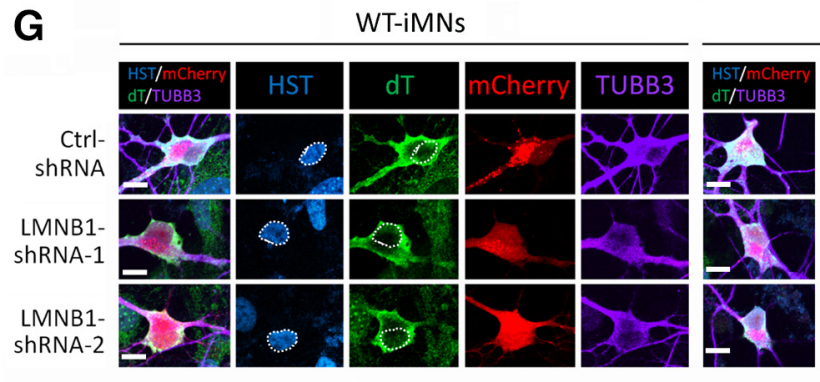

DYT1-iMNs
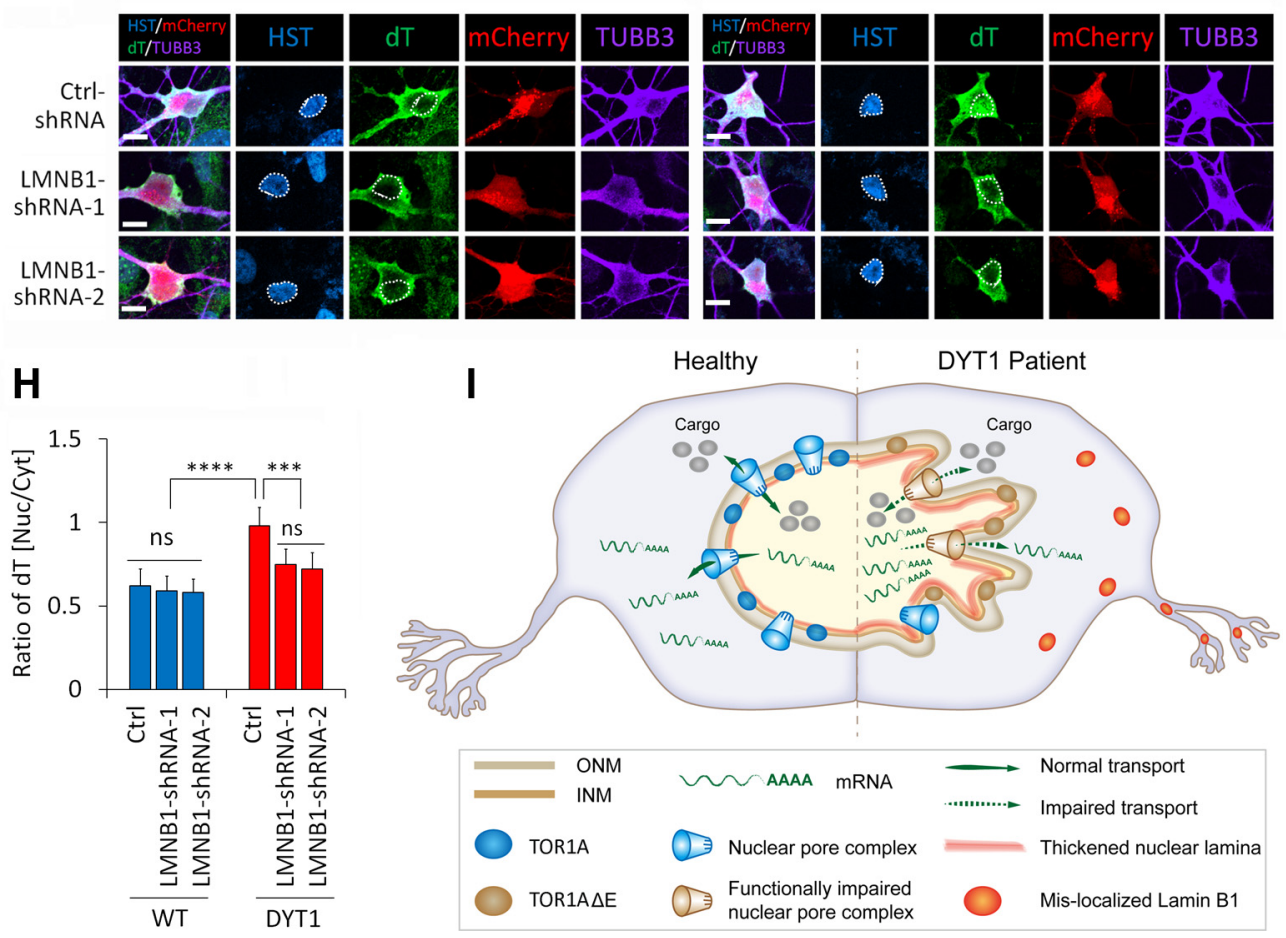

Figure 9. Phenotypic amelioration of DYT1 MNs via LMNB1 downregulation. $\boldsymbol{A}$, Western blottings showing shRNA-mediated downregulation of LMNB1. The single band of LMNB1 on the whole blotting membrane indicates the specificity of LMNB1 antibody. Actin serves as a loading control. $\boldsymbol{B}$, Representative confocal images of iMNs with the indicated shRNAs (mCherry + ) at 3 wpi. Scale bar: $25 \mu \mathrm{m}$. $\boldsymbol{C}$, Percentage of iMNs (mCherry + ) with mislocalized LMNB1 in $\boldsymbol{B}$ [n (neurons) $>200$ for each condition from triplicates; ns, not significant; $* p=0.032$, $* * * * p<0.0001$, ANOVA]. D, Quantitative analysis of neurite length of iMNs (mCherry + ) at 2 wpi under the indicated conditions $[n$ (neurons) $>100$ for each sample from triplicates; ns, not significant; $* * p=0.0074, * * * p=0.00017$ (vs DYT1-Ctrl), ANOVA; $* * * * p=1.26 \mathrm{E}-05$ (WT-Ctrl vs DYT1-Ctrl), $t$ test]. $\boldsymbol{E}$, Neurite number of the primary branches in iMNs (mCherry + ) at 4 wpi [ $n$ (neurons) $>100$ each sample from triplicates; ns, not significant; $* * * p<0.00043, * * * * p=7.42 \mathrm{E}-05$ (vs DYT1-Ctrl), ANOVA; $* * * * p=2.833 \mathrm{E}-15$ (WT-Ctrl vs DYT1-Ctrl), $t$ test]. $\boldsymbol{F}$, Percentage of DYT1-iMNs with abnormal nuclear morphology at 3 wpi ( $n=104$ for DYT1, $n=98$ for Ctrl-shNRA, $n=72$ for LMNB1-shRNA1 and $n=69$ for LMNB1-shNRA2 from triplicates; ns, not significant; $* * * * p=5.34 \mathrm{E}-08$, ANOVA). $\mathbf{G}$, Representative confocal images of FISH assay of iMNs at 3 wpi. shRNA-expressing cells are identified by the co-expressed mCherry. Dotted lines highlight nuclei. Scale bar: $10 \mu \mathrm{m}$. $\boldsymbol{H}$, Quantification of oligo-dT signal distribution in $\boldsymbol{G}[n$ (neurons) $>100$ for each sample from triplicates; ns, not significant; $* * * p=0.00063$, ANOVA; $* * * * p=5.34 \mathrm{E}-09$ (WT-Ctrl vs DYT1-Ctrl), $t$ test]. I, A working model for DYT1 pathogenesis. The healthy MNs have normal nuclear morphology and NCT activity. In DYT1 MNs, LMNB1 


\section{Discussion}

Induced human neurons as novel models for DYT1 dystonia Most cases of DYT1 dystonia are caused by a single amino acid deletion in TOR1A under a heterozygous background. Interestingly, mice with the identical Torla mutation as a heterozygote failed to show any pathologic phenotypes (Goodchild et al., 2005), suggesting that species-specific differences may play a role in DYT1 pathology. Nonetheless, the limited access to patient neurons has greatly impeded the progress of DYT1 dystonia research. In this study, we generated DYT1 MNs via two strategies: direct conversion from patient fibroblasts and iPSC-based reprogramming and differentiation. These neurons are in the same genetic background as human patients and maintain endogenous levels of TOR1A expression. As such, they provide an unprecedented in vitro cellular model for dystonia research.

DYT1 MNs from both strategies show similar phenotypes including delayed neuronal development, abnormal nuclear morphology, and disrupted mRNA nuclear export and protein nuclear transport. Unlike iPSCs, which are epigenetically reset to an embryonic state (Lapasset et al., 2011; Soria-Valles and López-Otín, 2016), directly reprogramed neurons from donors retain features associated with age and disease (Mertens et al., 2015; Tang et al., 2017). We consistently find that DYT1-diMNs from fibroblasts exhibit more severe defects than DYT1-iMNs from iPSCs in neurite outgrowth, nuclear morphology, and NCT. However, the iPSC-based strategy has several unique advantages: both the yield and the purity of iMNs are much higher than diMNs, and the culture time to obtain mature neurons is also shorter. Therefore, iMNs are suitable for experiments requiring a large amount of samples, whereas diMNs can provide aging-relevant neurons for single cell analysis. Both strategies should be valuable in DYT1 dystonia research.

Our study further provides evidence that lower MNs are implicated in the pathogenesis of dystonia. We reveal for the first time that LMNB1 is upregulated and mislocalized in DYT1 MNs. Most interestingly, downregulation of LMNB1 can significantly improve development, NE morphology, and mRNA nuclear export of DYT1 MNs. Our results support a model that upregulated LMNB1 causes thickening of the nuclear lamina, thereby leading to abnormal nuclear morphology and impaired NCT in DYT1 MNs (Fig. 9I). Progressive LMNB1 accumulation and subcellular mislocalization are key pathologic features of DYT1 MNs and may constitute a novel molecular target for intervention.

\section{Increased nuclear LMNB1 causes abnormal NE morphology} Nuclear LMNB1 is an essential scaffolding component of the lamina meshwork beneath the NE. It plays important roles in determining nuclear morphology, DNA regulation, and gene expression (Broers et al., 2006; Turgay et al., 2017). Overexpression of LMNB1 is shown to increase nuclear rigidity (Ferrera et al., 2014). This is consistent with our result that LMNB1 overexpression can disrupt nuclear morphology in fibroblasts. An abnormal extra copy of LMNB1 gene causes autosomal dominant leukodystrophy (ADLD) characterized by loss of myelin in the brain and the spinal cord, leading to movement problems (Lin et al., 2014; Padiath, 2016; Köhler et al., 2018). Consistently, we find that LMNB1 expression is

$\leftarrow$

upregulation leads to thickened nuclear lamina, cytoplasmic localization, abnormal nuclear morphology, and impaired NCT, which may subsequently lead to mislocalization of mRNAs and proteins and dysfunction of neurons. obviously increased in DYT1 MNs, and the NE morphology is abnormal at both light-microscope and electron-microscope levels. Downregulation of LMNB1 in DYT1 MNs can alleviate the defects in neuron development, NE morphology, and mRNA nuclear export, suggesting that increased LMNB1 contributes to these abnormalities in DYT1 neurons.

Despite intensive efforts examining the NE structure by TEM, we failed to detect bleb-like structures in DYT1 MNs. Such a result is in sharp contrast to what was observed in Drosophila with torsin mutant or in mice with Torla knock-out or Tor $1 a^{\Delta E / \Delta E}$ (Goodchild et al., 2005; Jokhi et al., 2013; Tanabe et al., 2016). This discrepancy may be caused by species differences and/or functional redundancy between different human Torsin genes in NE morphology maintenance (Laudermilch et al., 2016; Li et al., 2020). Only one torsin gene exists in Drosophila whereas there are four Torsin genes in humans. Additionally, all human patients are heterozygous with one remaining WT copy of TOR1A gene that may also diminish such severe abnormalities as nuclear blebs. On the other hand, the formation of these bleb-like structures is cell cycle dependent (Rampello et al., 2020) and developmentally regulated (Tanabe et al., 2016). The bleb-like structures could occur transiently before neuronal differentiation but be quickly resolved at later stages, at which the samples we have examined. As such, it would be minor, if any, for a potential contribution of a defective NE-budding mechanism (Speese et al., 2012) to the impaired mRNA export in differentiated DYT1 MNs. In contrast, our results rather suggest that an impaired NPC-mediated transport pathway is the major causing factor for the defects observed in human DYT1 MNs.

\section{Defective nuclear pore biogenesis and impaired NCT}

The NE and embedded NPCs play critical roles in regulating NCT of both transcripts and proteins (Köhler and Hurt, 2007; Grünwald et al., 2011; Katahira, 2015; Beck and Hurt, 2017). Accumulating evidence indicates that Torsin and its cofactor system could be involved in the nuclear pore biogenesis or homeostasis (Laudermilch et al., 2016; Chase et al., 2017; Rampello et al., 2020). In Torsin-deficient cells, one distinguished cellular feature is the "blebbing" structures at perinuclear space as discussed above. This phenotype has been observed in neural tissues of DYT1 mouse models (Tor1a knock-out or Tor $1 a^{\Delta E / \Delta E}$ overexpression; Goodchild et al., 2005; Tanabe et al., 2016) and mammalian cells with combined knock-outs of multiple Torsins (Laudermilch et al., 2016; Rampello et al., 2020). Interestingly, a subset of FG-Nups have been identified at the electron-dense base of NE blebs and the size of this density is similar to mature NPCs (Laudermilch et al., 2016). By using Torsin deletion HeLa cell line (4TorKO with deletion of all four Torsin genes TOR1A, TOR1B, TOR2A, and TOR3A), Rampello et al. (2020) recently demonstrated that Torsin ATPase deficiency leads to defects in nuclear pore biogenesis. Supporting such a conclusion, we find significantly fewer NPC structures in DYT1 MNs than controls.

Although the precise mechanism of NPC assembly is not fully elucidated, two different assembly pathways have been proposed. One pathway occurs postmitotically while NE reforms and the other occurs during interphase (Doucet et al., 2010). The model of an inside-out extrusion of the NE reveals a multistep process of the nuclear pore assembly during interphase (Otsuka, 2016). This assembly starts from the evaginations of inner nuclear membrane as intermediates, which initially contain the nuclear and cytoplasmic ring component of the NPC. While protruding toward the outer nuclear membrane, several subcomplexes of the NPC will be assembled, and then the inner and outer nuclear 
membranes are fused together. Some components, such as the cytoplasmic filament NUP358, are added to the NPC after this fusion event, completing the assembly of mature NPCs from intermediates (Otsuka, 2016). Recent reports suggest that Torsin plays an important role in adding NUP358 to form mature NPCs in the interphase insertion pathway. During postnatal maturation, Torla-null mouse neurons develop mislocalized and dysfunctional NPCs that lack NUP358 (Pappas et al., 2018). In Torsin-deficient cells, NUP358 is underrepresented relative to FG-nucleoporins in nuclear envelops (Rampello et al., 2020). In current study, our measurement of the nuclear pore size under TEM fails to find significant differences between DYT1 MNs and controls. A caveat is that the resolution of TEM may not detect the diameter differences between immature (without NUP358) and mature NPCs (with NUP358). Given that $~ 50 \%$ of all NPCs are installed via a postmitotic insertion pathway (Doucet et al., 2010), it may not be surprising that the nuclear pore size is relatively normal in mature DYT1 neurons, a developmental stage at which Torsin has less of an effect on NPC assembly. Nonetheless, the abnormally assembled NPCs at early developmental stages may cause persistent defects on neuronal nuclear pores and NCT (Pappas et al., 2018).

\section{References}

Albanese A, Bhatia K, Bressman SB, Delong MR, Fahn S, Fung VS, Hallett M, Jankovic J, Jinnah HA, Klein C, Lang AE, Mink JW, Teller JK (2013) Phenomenology and classification of dystonia: a consensus update. Mov Disord 28:863-873.

Arber S, Han B, Mendelsohn M, Smith M, Jessell TM, Sockanathan S (1999) Requirement for the homeobox gene $\mathrm{Hb} 9$ in the consolidation of motor neuron identity. Neuron 23:659-674.

Beck M, Hurt E (2017) The nuclear pore complex: understanding its function through structural insight. Nat Rev Mol Cell Biol 18:73-89.

Broers JL, Ramaekers FC, Bonne G, Yaou RB, Hutchison CJ (2006) Nuclear lamins: laminopathies and their role in premature ageing. Physiol Rev 86:967-1008.

Burke B, Stewart CL (2013) The nuclear lamins: flexibility in function. Nat Rev Mol Cell Biol 14:13-24.

Capell BC, Collins FS (2006) Human laminopathies: nuclei gone genetically awry. Nat Rev Genet 7:940-952.

Charlesworth G, Bhatia KP, Wood NW (2013) The genetics of dystonia: new twists in an old tale. Brain 136:2017-2037.

Chase AR, Laudermilch E, Schlieker C (2017) Torsin ATPases: harnessing dynamic instability for function. Front Mol Biosci 4:29.

Dauer W (2014) Inherited isolated dystonia: clinical genetics and gene function. Neurotherapeutics 11:807-816.

Ding B (2015) Gene expression in maturing neurons: regulatory mechanisms and related neurodevelopmental disorders. Sheng Li Xue Bao 67:113133.

Ding B, Kilpatrick DL (2013) Lentiviral vector production, titration, and transduction of primary neurons. Methods Mol Biol 1018:119-131.

Ding B, Wang W, Selvakumar T, Xi HS, Zhu H, Chow CW, Horton JD, Gronostajski RM, Kilpatrick DL (2013) Temporal regulation of nuclear factor one occupancy by calcineurin/NFAT governs a voltage-sensitive developmental switch in late maturing neurons. J Neurosci 33:28602872.

Ding B, Cave JW, Dobner PR, Mullikin-Kilpatrick D, Bartzokis M, Zhu H, Chow CW, Gronostajski RM, Kilpatrick DL (2016) Reciprocal autoregulation by NFI occupancy and ETV1 promotes the developmental expression of dendrite-synapse genes in cerebellar granule neurons. Mol Biol Cell 27:1488-1499.

Ding B, Dobner PR, Mullikin-Kilpatrick D, Wang W, Zhu H, Chow CW, Cave JW, Gronostajski RM, Kilpatrick DL (2018) BDNF activates an NFI-dependent neurodevelopmental timing program by sequestering NFATc4. Mol Biol Cell 29:975-987.

Ding B, Akter M, Zhang CL (2020) Differential influence of sample sex and neuronal maturation on mRNA and protein transport in induced human neurons. Front Mol Neurosci 13:46.
Doucet CM, Talamas JA, Hetzer MW (2010) Cell cycle-dependent differences in nuclear pore complex assembly in metazoa. Cell 141:1030-1041.

Ferrera D, Canale C, Marotta R, Mazzaro N, Gritti M, Mazzanti M, Capellari S, Cortelli P, Gasparini L (2014) Lamin B1 overexpression increases nuclear rigidity in autosomal dominant leukodystrophy fibroblasts. FASEB J 28:3906-3918.

Fremont R, Tewari A, Angueyra C, Khodakhah K (2017) A role for cerebellum in the hereditary dystonia DYT1. Elife 6:e22775.

Goodchild RE, Kim CE, Dauer WT (2005) Loss of the dystonia-associated protein torsinA selectively disrupts the neuronal nuclear envelope. Neuron 48:923-932.

Granata A, Watson R, Collinson LM, Schiavo G, Warner TT (2008) The dystonia-associated protein torsinA modulates synaptic vesicle recycling. J Biol Chem 283:7568-7579.

Granata A, Schiavo G, Warner TT (2009) TorsinA and dystonia: from nuclear envelope to synapse. J Neurochem 109:1596-1609.

Grünwald D, Singer RH, Rout M (2011) Nuclear export dynamics of RNAprotein complexes. Nature 475:333-341.

Hewett JW, Zeng J, Niland BP, Bragg DC, Breakefield XO (2006) Dystoniacausing mutant torsin A inhibits cell adhesion and neurite extension through interference with cytoskeletal dynamics. Neurobiol Dis 22:98111.

Ip CW, Isaias IU, Kusche-Tekin BB, Klein D, Groh J, O'Leary A, Knorr S, Higuchi T, Koprich JB, Brotchie JM, Toyka KV, Reif A, Volkmann J (2016) Torla +/- mice develop dystonia-like movements via a striatal dopaminergic dysregulation triggered by peripheral nerve injury. Acta Neuropathol Commun 4:108.

Jokhi V, Ashley J, Nunnari J, Noma A, Ito N, Wakabayashi-Ito N, Moore MJ, Budnik V (2013) Torsin mediates primary envelopment of large ribonucleoprotein granules at the nuclear envelope. Cell Rep 3:988-995.

Katahira J (2015) Nuclear export of messenger RNA. Genes (Basel) 6:163184.

Köhler A, Hurt E (2007) Exporting RNA from the nucleus to the cytoplasm. Nat Rev Mol Cell Biol 8:761-773.

Köhler W, Curiel J, Vanderver A (2018) Adulthood leukodystrophies. Nat Rev Neurol 14:94-105.

Lapasset L, Milhavet O, Prieur A, Besnard E, Babled A, Ait-Hamou N, Leschik J, Pellestor F, Ramirez JM, De Vos J, Lehmann S, Lemaitre JM (2011) Rejuvenating senescent and centenarian human cells by reprogramming through the pluripotent state. Genes Dev 25:2248-2253.

Laudermilch E, Schlieker C (2016) Torsin ATPases: structural insights and functional perspectives. Curr Opin Cell Biol 40:1-7.

Laudermilch E, Tsai PL, Graham M, Turner E, Zhao C, Schlieker C (2016) Dissecting Torsin/cofactor function at the nuclear envelope: a genetic study. Mol Biol Cell 27:3964-3971.

Li J, Liang CC, Pappas SS, Dauer WT (2020) TorsinB overexpression prevents abnormal twisting in DYT1 dystonia mouse models. Elife 9:e54285.

Li Y, Hassinger L, Thomson T, Ding B, Ashley J, Hassinger W, Budnik V (2016) Lamin mutations accelerate aging via defective export of mitochondrial mRNAs through nuclear envelope budding. Curr Biol 26:2052-2059.

Lin ST, Heng MY, Ptáček LJ, Fu YH (2014) Regulation of myelination in the central nervous system by nuclear lamin B1 and non-coding RNAs. Transl Neurodegener 3:4.

Liu ML, Zang T, Zou Y, Chang JC, Gibson JR, Huber KM, Zhang CL (2013) Small molecules enable neurogenin 2 to efficiently convert human fibroblasts into cholinergic neurons. Nat Commun 4:2183.

Liu ML, Zang T, Zhang CL (2016) Direct lineage reprogramming reveals disease-specific phenotypes of motor neurons from human ALS patients. Cell Rep 14:115-128.

Lohmann K, Klein C (2013) Genetics of dystonia: what's known? What's new? What's next? Mov Disord 28:899-905.

Maltese M, Stanic J, Tassone A, Sciamanna G, Ponterio G, Vanni V, Martella G, Imbriani P, Bonsi P, Mercuri NB, Gardoni F, Pisani A (2018) Early structural and functional plasticity alterations in a susceptibility period of DYT1 dystonia mouse striatum. Elife 7:e33331.

Mertens J, Paquola AC, Ku M, Hatch E, Böhnke L, Ladjevardi S, McGrath S, Campbell B, Lee H, Herdy JR, Goncalves JT, Toda T, Kim Y, Winkler J, Yao J, Hetzer MW, Gage FH (2015) Directly reprogrammed human neurons retain aging-associated transcriptomic signatures and reveal agerelated nucleocytoplasmic defects. Cell Stem Cell 17:705-718. 
Naismith TV, Heuser JE, Breakefield XO, Hanson PI (2004) TorsinA in the nuclear envelope. Proc Natl Acad Sci USA 101:7612-7617.

Nery FC, Zeng J, Niland BP, Hewett J, Farley J, Irimia D, Li Y, Wiche G, Sonnenberg A, Breakefield XO (2008) TorsinA binds the KASH domain of nesprins and participates in linkage between nuclear envelope and cytoskeleton. J Cell Sci 121:3476-3486.

Otsuka S, Huy Bui K, Schorb M, Hossain JM, Politi AZ, Koch B, Eltsov M, Beck M, Ellenberg J (2016) Nuclear pore assembly proceeds by an insideout extrusion of the nuclear envelope. Elife 5:e19071.

Ozelius LJ, Hewett JW, Page CE, Bressman SB, Kramer PL, Shalish C, de Leon D, Brin MF, Raymond D, Corey DP, Fahn S, Risch NJ, Buckler AJ, Gusella JF, Breakefield XO (1997) The early-onset torsion dystonia gene (DYT1) encodes an ATP-binding protein. Nat Genet 17:40-48.

Packard M, Jokhi V, Ding B, Ruiz-Cañada C, Ashley J, Budnik V (2015) Nucleus to synapse nesprin1 railroad tracks direct synapse maturation through RNA localization. Neuron 86:1015-1028.

Padiath QS (2016) Lamin B1 mediated demyelination: linking lamins, lipids and leukodystrophies. Nucleus 7:547-553.

Pappas SS, Liang CC, Kim S, Rivera CO, Dauer WT (2018) TorsinA dysfunction causes persistent neuronal nuclear pore defects. Hum Mol Genet 27:407-420.

Pelossof R, Fairchild L, Huang CH, Widmer C, Sreedharan VT, Sinha N, Lai DY, Guan Y, Premsrirut PK, Tschaharganeh DF, Hoffmann T, Thapar V, Xiang Q, Garippa RJ, Rätsch G, Zuber J, Lowe SW, Leslie CS, Fellmann C (2017) Prediction of potent shRNAs with a sequential classification algorithm. Nat Biotechnol 35:350-353.

Rampello AJ, Laudermilch E, Vishnoi N, Prophet SM, Shao L, Zhao C, Lusk CP, Schlieker C (2020) Torsin ATPase deficiency leads to defects in nuclear pore biogenesis and sequestration of MLF2. J Cell Biol 219: e201910185.

Rostasy K, Augood SJ, Hewett JW, Leung JC, Sasaki H, Ozelius LJ, Ramesh V, Standaert DG, Breakefield XO, Hedreen JC (2003) TorsinA protein and neuropathology in early onset generalized dystonia with GAG deletion. Neurobiol Dis 12:11-24.

Sepehrimanesh M, Ding B (2020) Generation and optimization of highly pure motor neurons from human induced pluripotent stem cells via lentiviral delivery of transcription factors. Am J Physiol Cell Physiol 319: C771-C780

Shah P, Wolf K, Lammerding J (2017) Bursting the bubble - nuclear envelope rupture as a path to genomic instability? Trends Cell Biol 27:546-555.
Soria-Valles C, López-Otín C (2016) iPSCs: on the road to reprogramming aging. Trends Mol Med 22:713-724.

Speese SD, Ashley J, Jokhi V, Nunnari J, Barria R, Li Y, Ataman B, Koon A, Chang Y-T, Li Q, Moore MJ, Budnik V (2012) Nuclear envelope budding enables large ribonucleoprotein particle export during synaptic Wnt signaling. Cell 149:832-846.

Takahashi K, Okita K, Nakagawa M, Yamanaka S (2007) Induction of pluripotent stem cells from fibroblast cultures. Nat Protoc 2:3081-3089.

Tanabe LM, Liang CC, Dauer WT (2016) Neuronal nuclear membrane budding occurs during a developmental window modulated by torsin paralogs. Cell Rep 16:3322-3333.

Tang Y, Liu ML, Zang T, Zhang CL (2017) Direct reprogramming rather than iPSC-based reprogramming maintains aging hallmarks in human motor neurons. Front Mol Neurosci 10:359.

Tatli M, Medalia O (2018) Insight into the functional organization of nuclear lamins in health and disease. Curr Opin Cell Biol 54:72-79.

Tewari A, Fremont R, Khodakhah K (2017) It's not just the basal ganglia: cerebellum as a target for dystonia therapeutics. Mov Disord 32:1537-1545.

Torres GE, Sweeney AL, Beaulieu JM, Shashidharan P, Caron MG (2004) Effect of torsinA on membrane proteins reveals a loss of function and a dominant-negative phenotype of the dystonia-associated DeltaE-torsinA mutant. Proc Natl Acad Sci USA 101:15650-15655.

Turgay Y, Eibauer M, Goldman AE, Shimi T, Khayat M, Ben-Harush K, Dubrovsky-Gaupp A, Sapra KT, Goldman RD, Medalia O (2017) The molecular architecture of lamins in somatic cells. Nature 543:261-264.

Vander Heyden AB, Naismith TV, Snapp EL, Hanson PI (2011) Static retention of the lumenal monotopic membrane protein torsinA in the endoplasmic reticulum. EMBO J 30:3217-3231.

VanGompel MJ, Nguyen KC, Hall DH, Dauer WT, Rose LS (2015) A novel function for the Caenorhabditis elegans torsin OOC-5 in nucleoporin localization and nuclear import. Mol Biol Cell 26:1752-1763.

Wilson KL, Foisner R (2010) Lamin-binding proteins. Cold Spring Harb Perspect Biol 2:a000554.

Yang J, Tang Y, Liu H, Guo F, Ni J, Le W (2014) Suppression of histone deacetylation promotes the differentiation of human pluripotent stem cells towards neural progenitor cells. BMC Biol 12:95.

Zhao C, Brown RS, Chase AR, Eisele MR, Schlieker C (2013) Regulation of torsin ATPases by LAP1 and LULL1. Proc Natl Acad Sci USA 110: E1545-E1554. 